\title{
A New Family of the $\lambda$-Generalized Hurwitz-Lerch Zeta Functions with Applications
}

\author{
H. M. Srivastava* \\ Department of Mathematics and Statistics, University of Victoria, Victoria, British Columbia V8W 3R4, Canada
}

Received: 3 Jul. 2013, Revised: 5 Oct. 2013, Accepted: 6 Oct. 2013

Published online: 1 Jul. 2014

\begin{abstract}
Motivated largely by a number of recent investigations, we introduce and investigate the various properties of a certain new family of the $\lambda$-generalized Hurwitz-Lerch zeta functions. We derive many potentially useful results involving these $\lambda$-generalized Hurwitz-Lerch zeta functions including (for example) their partial differential equations, new series and Mellin-Barnes type contour integral representations (which are associated with Fox's $H$-function) and several other summation formulas. We discuss their potential application in Number Theory by appropriately constructing a seemingly novel continuous analogue of Lippert's Hurwitz measure. We also consider some other statistical applications of the family of the $\lambda$-generalized Hurwitz-Lerch zeta functions in probability distribution theory.
\end{abstract}

Keywords: Hurwitz-Lerch zeta function; Analytic Number Theory; $\lambda$-Generalized Hurwitz-Lerch zeta functions; Derivative properties; Partial differential equations; Series representations; Mellin-Barnes contour integral representations; Generalized Hurwitz meausure; Probability density functions.

2010 Mathematics Subject Classification. Primary 11M06, 11M35, 33B15; Secondary 11B68, 33C65, 33C90.

\section{Introduction and Definitions}

Throughout our present investigation, we use the following standard notations:

$$
\mathbb{N}:=\{1,2,3, \cdots\}, \quad \mathbb{N}_{0}:=\{0,1,2,3, \cdots\}=\mathbb{N} \cup\{0\}
$$

and

$$
\mathbb{Z}^{-}:=\{-1,-2,-3, \cdots\}=\mathbb{Z}_{0}^{-} \backslash\{0\} .
$$

Also, as usual, $\mathbb{Z}$ denotes the set of integers, $\mathbb{R}$ denotes the set of real numbers, $\mathbb{R}^{+}$denotes the set of positive numbers and $\mathbb{C}$ denotes the set of complex numbers.

One of the fundamentally important higher transcendental functions of Analytic Number Theory is the familiar general Hurwitz-Lerch Zeta function $\Phi(z, s, a)$ defined by (see, for example, [5, p. 27. Eq. 1.11 (1)]; see also [26], [29, p. 121 et seq.] and [30, p. 194 et seq.])

$$
\Phi(z, s, a):=\sum_{n=0}^{\infty} \frac{z^{n}}{(n+a)^{s}}
$$

$\left(a \in \mathbb{C} \backslash \mathbb{Z}_{0}^{-} ; s \in \mathbb{C}\right.$ when $|z|<1 ; \Re(s)>1$ when $\left.|z|=1\right)$. It contains, as its special cases, not only the Riemann Zeta function $\zeta(s)$, the Hurwitz (or generalized) Zeta function $\zeta(s, a)$ and the Lerch Zeta function $\ell_{s}(\xi)$ defined by (see, for details, [5, Chapter I] and [29, Chapter 2])

$$
\begin{array}{r}
\zeta(s):=\sum_{n=1}^{\infty} \frac{1}{n^{s}}=\Phi(1, s, 1)=\zeta(s, 1) \\
(\Re(s)>1), \\
\zeta(s, a):=\sum_{n=0}^{\infty} \frac{1}{(n+a)^{s}}=\Phi(1, s, a) \\
\left(\Re(s)>1 ; a \in \mathbb{C} \backslash \mathbb{Z}_{0}^{-}\right)
\end{array}
$$

and

$$
\begin{array}{r}
\ell_{s}(\xi):=\sum_{n=0}^{\infty} \frac{e^{2 n \pi \mathrm{i} \xi}}{(n+1)^{s}}=\Phi\left(e^{2 \pi \mathrm{i} \xi}, s, 1\right) \\
(\Re(s)>1 ; \xi \in \mathbb{R}),
\end{array}
$$

respectively, but also such other important functions of Analytic Number Theory as the Polylogarithmic function (or de Jonquière's function) $\mathrm{Li}_{s}(z)$ :

$$
\operatorname{Li}_{s}(z):=\sum_{n=1}^{\infty} \frac{z^{n}}{n^{s}}=z \Phi(z, s, 1)
$$

\footnotetext{
*Corresponding author e-mail: harimsri@math.uvic.ca
} 
$(s \in \mathbb{C} \quad$ when $|z|<1 ; \Re(s)>1$ when $|z|=1)$ and the Lipschitz-Lerch Zeta function $\phi(\xi, a, s)$ (see [29, p. 122, Equation 2.5 (11)]):

$$
\phi(\xi, s, a):=\sum_{n=0}^{\infty} \frac{e^{2 n \pi \mathrm{i} \xi}}{(n+a)^{s}}=\Phi\left(e^{2 \pi \mathrm{i} \xi}, s, a\right)
$$

$\left(a \in \mathbb{C} \backslash \mathbb{Z}_{0}^{-} ; \Re(s)>0 \quad\right.$ when $\quad \xi \in \mathbb{R} \backslash \mathbb{Z} ; \Re(s)>1$ when $\left.\xi \in \mathbb{Z}\right)$, which was first studied by Rudolf Lipschitz (1832-1903) and Matyáš Lerch (1860-1922) in connection with Dirichlet's famous theorem on primes in arithmetic progressions (see also [27, Section 5]). Indeed, just as its aforementioned special cases $\zeta(s)$ and $\zeta(s, a)$, the Hurwitz-Lerch Zeta function $\Phi(z, s, a)$ defined by (1.7) can be continued meromorphically to the whole complex $s$-plane, except for a simple pole at $s=1$ with its residue 1. It is also known that [5, p. 27, Equation 1.11 (3)]

$$
\begin{aligned}
\Phi(z, s, a) & =\frac{1}{\Gamma(s)} \int_{0}^{\infty} \frac{t^{s-1} e^{-a t}}{1-z e^{-t}} \mathrm{~d} t \\
& =\frac{1}{\Gamma(s)} \int_{0}^{\infty} \frac{t^{s-1} e^{-(a-1) t}}{e^{t}-z} \mathrm{~d} t
\end{aligned}
$$

$(\mathfrak{R}(a)>0 ; \mathfrak{R}(s)>0$ when $|z| \leqq 1(z \neq 1) ; \mathfrak{R}(s)>1$ when $z=1)$.

Recently, Srivastava et al. [36] introduced and systematically studied various properties and results involving a natural multiparameter extension and generalization of the Hurwitz-Lerch zeta function $\Phi(z, s, a)$ defined by (1.7) (see also [27] and [32]). In order to recall their definition (which was motivated essentially by the earlier works of Goyal and Laddha [10], Lin and Srivastava [15], Garg et al. [8], and other authors), each of the following notations will be employed:

$$
\nabla^{*}:=\left(\prod_{j=1}^{p} \rho_{j}^{-\rho_{j}}\right) \cdot\left(\prod_{j=1}^{q} \sigma_{j}^{\sigma_{j}}\right)
$$

and

$\Delta:=\sum_{j=1}^{q} \sigma_{j}-\sum_{j=1}^{p} \rho_{j} \quad, \quad \Xi:=s+\sum_{j=1}^{q} \mu_{j}-\sum_{j=1}^{p} \lambda_{j}+\frac{p-q}{2}$.

The extended Hurwitz-Lerch zeta function

$$
\Phi_{\lambda_{1}, \cdots, \lambda_{p} ; \mu_{1}, \cdots, \mu_{q}}^{\left(\rho_{1}, \cdots, \rho_{p}, \sigma_{1}, \cdots, \sigma_{q}\right)}(z, s, a)
$$

is then defined by [36, p. 503, Equation (6.2)] (see also [27] and [32])

$$
\begin{gathered}
\Phi_{\lambda_{1}, \cdots, \lambda_{p}, \mu_{1}, \cdots, \mu_{q}}^{\left(\rho_{1}, \cdots, \rho_{p}, \sigma_{1}, \cdots, \sigma_{q}\right)}(z, s, a) \\
:=\sum_{n=0}^{\infty} \frac{\prod_{j=1}^{p}\left(\lambda_{j}\right)_{n \rho_{j}}}{n ! \cdot \prod_{j=1}^{q}\left(\mu_{j}\right)_{n \sigma_{j}}} \frac{z^{n}}{(n+a)^{s}}
\end{gathered}
$$

$$
\begin{gathered}
\left(p, q \in \mathbb{N}_{0} ; \lambda_{j} \in \mathbb{C}(j=1, \cdots, p) ; a, \mu_{j} \in \mathbb{C} \backslash Z_{0}^{-}(j=1, \cdots, q) ;\right. \\
\rho_{j}, \sigma_{k} \in \mathbb{R}^{+}(j=1, \cdots, p ; k=1, \cdots, q) ; \\
\Delta>-1 \text { when } s, z \in \mathbb{C} ; \\
\Delta=-1 \text { and } s \in \mathbb{C} \text { when }|z|<\nabla^{*} ; \\
\left.\Delta=-1 \text { and } \mathfrak{R}(\Xi)>\frac{1}{2} \text { when }|z|=\nabla^{*}\right),
\end{gathered}
$$

where $(\lambda)_{v}(\lambda, v \in \mathbb{C})$ denotes the Pochhammer symbol (or the shifted factorial) which is defined, in terms of the familiar Gamma function, by

$$
(\lambda)_{v}:=\frac{\Gamma(\lambda+v)}{\Gamma(\lambda)}= \begin{cases}1 & (v=0 ; \lambda \in \mathbb{C} \backslash\{0\}) \\ \lambda(\lambda+1) \cdots(\lambda+n-1) & (v=n \in \mathbb{N} ; \lambda \in \mathbb{C}),\end{cases}
$$

it being understood conventionally that $(0)_{0}:=1$ and assumed tacitly that the above $\Gamma$-quotient exists. In terms of the extended Hurwitz-Lerch zeta function defined by (1.10), the following unification and generalization of several known integral representations stemming from (1.7) was given by Srivastava et al. [36] (see also [28, Theorem 6] for a more general sum-integral representation formula):

$$
\begin{gathered}
\Phi_{\lambda_{1}, \cdots, \lambda_{p} ; \mu_{1}, \cdots, \mu_{q}}^{\left(\rho_{1}, \cdots, \rho_{p}, \sigma_{1}, \cdots, \sigma_{q}\right)}(z, s, a)=\frac{1}{\Gamma(s)} \int_{0}^{\infty} t^{s-1} e^{-a t} \\
\cdot{ }_{p} \Psi_{q}^{*}\left[\begin{array}{l}
\left(\lambda_{1}, \rho_{1}\right), \cdots,\left(\lambda_{p}, \rho_{p}\right) ; \\
\left(\mu_{1}, \sigma_{1}\right), \cdots,\left(\mu_{q}, \sigma_{q}\right) ;
\end{array}\right] \mathrm{e}-\mathrm{d} t \\
(\min \{\Re(a), \Re(s)\}>0),
\end{gathered}
$$

provided that the integral exists. Here, and in what follows, ${ }_{p} \Psi_{q}^{*} \quad$ or ${ }_{p} \Psi_{q} \quad\left(p, q \in \mathbb{N}_{0}\right)$ denotes the Fox-Wright function, which is a generalization of the familiar generalized hypergeometric function ${ }_{p} F_{q}\left(p, q \in \mathbb{N}_{0}\right)$, with $p$ numerator parameters $a_{1}, \cdots, a_{p}$ and $q$ denominator parameters $b_{1}, \cdots, b_{q}$ such that $a_{j} \in \mathbb{C}(j=1, \cdots, p) \quad$ and $\quad b_{j} \in \mathbb{C} \backslash \mathbb{Z}_{0}^{-}(j=1, \cdots, q)$, defined by (see, for details, [5, p. 183], [33, p. 21 et seq.] and [35, p. 50 et seq.]; see also [14, p. 56], [21, p. 30] and [31, p. 19])

$$
\begin{aligned}
{ }_{p} \Psi_{q}^{*}\left[\begin{array}{l}
\left(a_{1}, A_{1}\right), \cdots,\left(a_{p}, A_{p}\right) ; \\
\left(b_{1}, B_{1}\right), \cdots,\left(b_{q}, B_{q}\right) ;
\end{array}\right] \\
\quad:=\sum_{n=0}^{\infty} \frac{\left(a_{1}\right)_{A_{1} n} \cdots\left(a_{p}\right)_{A_{p} n}}{\left(b_{1}\right)_{B_{1} n} \cdots\left(b_{q}\right)_{B_{q} n}} \frac{z^{n}}{n !} \\
=\frac{\Gamma\left(b_{1}\right) \cdots \Gamma\left(b_{q}\right)}{\Gamma\left(a_{1}\right) \cdots \Gamma\left(a_{p}\right)}{ }_{p} \Psi_{q}\left[\begin{array}{l}
\left(a_{1}, A_{1}\right), \cdots,\left(a_{p}, A_{p}\right) ; \\
\left(b_{1}, B_{1}\right), \cdots,\left(b_{q}, B_{q}\right) ;
\end{array}\right] \\
\quad\left(A_{j}>0 \quad(j=1, \cdots, p) ; B_{j}>0\right. \\
\left.(j=1, \cdots, q) ; 1+\sum_{j=1}^{q} B_{j}-\sum_{j=1}^{p} A_{j} \geq 0\right),
\end{aligned}
$$


where the equality in the convergence condition holds true for suitably bounded values of $|z|$ given by

$$
|z|<\nabla:=\left(\prod_{j=1}^{p} A_{j}^{-A_{j}}\right) \cdot\left(\prod_{j=1}^{q} B_{j}^{B_{j}}\right)
$$

Definition 1. By suitably modifying this last integral representation formula (1.11), we now introduce and investigate the various properties of a significantly more general class of Hurwitz-Lerch zeta type functions defined by

$$
\begin{aligned}
\Phi_{\lambda_{1}, \cdots, \lambda_{p} ; \mu_{1}, \cdots, \mu_{q}}^{\left(\rho_{1}, \cdots, \rho_{p}, \sigma_{1}, \cdots, \sigma_{q}\right)}(z, s, a ; b, \lambda) & \frac{1}{\Gamma(s)} \int_{0}^{\infty} t^{s-1} \exp \left(-a t-\frac{b}{t^{\lambda}}\right) \\
& \left.\cdot{ }_{p} \Psi_{q}^{*}\left[\begin{array}{l}
\left(\lambda_{1}, \rho_{1}\right), \cdots,\left(\lambda_{p}, \rho_{p}\right) ; \\
\left(\mu_{1}, \sigma_{1}\right), \cdots,\left(\mu_{q}, \sigma_{q}\right) ;
\end{array}\right] \begin{array}{c}
-t \\
=
\end{array}\right] \mathrm{d} t, \\
& (\min \{\Re(a), \Re(s)\}>0 ; \Re(b) \geqq 0 ; \lambda \geqq 0),
\end{aligned}
$$

so that, obviously, we have the following relationship:

$$
\begin{aligned}
& \Phi_{\lambda_{1}, \cdots, \lambda_{p} ; \mu_{1}, \cdots, \mu_{q}}^{\left(\rho_{1}, \cdots, \rho_{p}, \sigma_{1}, \cdots, \sigma_{q}\right)}(z, s, a ; 0, \lambda) \\
& \quad=\Phi_{\lambda_{1}, \cdots, \lambda_{p} ; \mu_{1}, \cdots, \mu_{q}}^{\left(\rho_{1},,_{p}, \sigma_{1}\right)}(z, s, a) \\
& \quad=e^{b} \Phi_{\lambda_{1}, \cdots, \lambda_{p} ; \mu_{1}, \cdots, \mu_{q}}^{\left(\rho_{1}, \cdots, \rho_{p}, \sigma_{1}, \cdots, \sigma_{q}\right)}(z, s, a ; b, 0) .
\end{aligned}
$$

In its special case when

$$
p-1=q=0 \quad\left(\lambda_{1}=\mu ; \rho_{1}=1\right),
$$

the above definition (1.14) would reduce immediately to the following form:

$$
\begin{array}{r}
\Theta_{\mu}^{\lambda}(z, s, a ; b):=\frac{1}{\Gamma(s)} \int_{0}^{\infty} t^{s-1} \exp \left(-a t-\frac{b}{t^{\lambda}}\right) \\
\cdot\left(1-z e^{-t}\right)^{-\mu} \mathrm{d} t
\end{array}
$$

$(\min \{\Re(a), \Re(s)\}>0 ; \Re(b) \geqq 0 ; \lambda \geqq 0 ; \mu \in \mathbb{C})$,

where we have assumed further that

$$
\Re(s)>0 \quad \text { when } \quad b=0 \quad \text { and } \quad|z| \leqq 1 \quad(z \neq 1)
$$

or

$$
\Re(s-\mu)>0 \quad \text { when } \quad b=0 \quad \text { and } \quad z=1,
$$

provided, of course, that the integral in (1.16) exists. The function $\Theta_{\mu}^{\lambda}(z, s, a ; b)$ was introduced and studied by Raina and Chhajed [23, p. 90, Equation (1.6)] and (more recently) by Srivastava et al. [34].
Two interesting further special cases of the function $\Theta_{\mu}^{\lambda}(z, s, a ; b)$ are worthy of note here. First of all, for $b=0$, we find from the definition (1.16) that

$$
\begin{aligned}
\Theta_{\mu}^{\lambda}(z, s, a ; 0) & =\Phi_{\mu}^{*}(z, s, a) \\
& =\frac{1}{\Gamma(s)} \int_{0}^{\infty} \frac{t^{s-1} e^{-a t}}{\left(1-z e^{-t}\right)^{\mu}} \mathrm{d} t
\end{aligned}
$$

$(\Re(a)>0 ; \Re(s)>0$ when $|z| \leqq 1(z \neq 1) ; \Re(s-\mu)>0$ when $z=1)$, where the function $\Phi_{\mu}^{*}(z, s, a)$ defined by

$$
\Phi_{\mu}^{*}(z, s, a):=\sum_{n=0}^{\infty} \frac{(\mu)_{n}}{(a+n)^{s}} \frac{z^{n}}{n !}
$$

was studied by Goyal and Laddha [10, p. 100, Equation (1.5)]. As a matter of fact, in terms of the Riemann-Liouville fractional derivative operator $\mathscr{D}_{z}^{\mu}$ defined by (see, for example, [6, p. 181], [14, p. 70 et seq.] and [24])

$\mathscr{D}_{z}^{\mu}\{f(z)\}:= \begin{cases}\frac{1}{\Gamma(-\mu)} \int_{0}^{z}(z-t)^{-\mu-1} f(t) d t & (\mathfrak{R}(\mu)<0) \\ \frac{d^{m}}{d z^{m}}\left\{\mathscr{D}_{z}^{\mu-m}\{f(z)\}\right\} & (m-1 \leqq \Re(\mu)<m(m \in \mathbb{N})),\end{cases}$

it is easily seen from the series definitions in (1.1) and (1.18) that

$$
\Phi_{\mu}^{*}(z, s, a)=\frac{1}{\Gamma(\mu)} \mathscr{D}_{z}^{\mu-1}\left\{z^{\mu-1} \Phi(z, s, a)\right\}
$$

$$
(\Re(\mu)>0),
$$

which (as already remarked by Lin and Srivastava [15, p. 730]) exhibits the interesting (and useful) fact that the function $\Phi_{\mu}^{*}(z, s, a)$ is essentially a Riemann-Liouville fractional derivative of the classical Hurwitz-Lerch function $\Phi(z, s, a)$ (see also the closely-related investigations by Garg et al. [9] and Lin et al. [16]).

The other interesting special case of the function $\Theta_{\mu}^{\lambda}(z, s, a ; b)$ arises when we set $\lambda=\mu=1$ and $z=1$ in the definition (1.16). We thus find that

$$
\begin{aligned}
& \Theta_{1}^{1}(1, s, a ; b) \\
& \quad=\zeta_{b}(s, a) \\
& \quad:=\frac{1}{\Gamma(s)} \int_{0}^{\infty} \frac{t^{s-1} \exp \left(-a t-\frac{b}{t}\right)}{1-e^{-t}} \mathrm{~d} t
\end{aligned}
$$

where $\zeta_{b}(s, a)$ is the extended Hurwitz zeta function defined in [4, p. 308]. In fact, just as it is already pointed out in [18], the series representation (see [23, p. 91, Equation (2.1)]) given for the function $\Theta_{\mu}^{\lambda}(z, s, a ; b)$ in (1.16) is incorrect. Obvious further specializations in (1.17) and (1.20) would immediately relate these functions with the Riemann zeta function $\zeta(s)$ and the Hurwitz (or generalized) zeta function $\zeta(s, a)$ defined by 
(1.2) and (1.3), respectively.

Remark 1. In a series of recent papers, Bayad et al. (see [2], [3] and [7]) introduced and studied the so-called generalized Hurwitz-Lerch zeta function $\zeta(s, \mu ; a, z)$ of order $\mu$, which they defined by (cf. [3, p. 608, Equation (6)])

$$
\zeta(s, \mu ; a, z):=\frac{\Gamma(\mu)}{\Gamma(s)} \int_{0}^{\infty} \frac{t^{s-1} e^{-a t}}{\left(1-z e^{-t}\right)^{\mu}} \mathrm{d} t
$$

$$
\begin{gathered}
(\mathfrak{R}(a)>0 ; \mathfrak{R}(s)>0 \text { when }|z| \leqq 1 \quad(z \neq 1) ; \\
\Re(s-\mu)>0 \text { when } z=1)
\end{gathered}
$$

or, equivalently, by (cf. [3, p. 608, Equation (7)])

$$
\zeta(s, \mu ; a, z):=\sum_{n=0}^{\infty} \frac{\Gamma(\mu+n)}{n !} \frac{z^{n}}{(a+n)^{s}} .
$$

By comparing the definitions (1.18) and (1.22), it is easily observed that

$$
\zeta(s, \mu ; a, z)=\Gamma(\mu) \cdot \Phi_{\mu}^{*}(z, s, a),
$$

that is, that

$$
\Phi_{\mu}^{*}(z, s, a)=\frac{1}{\Gamma(\mu)} \zeta(s, \mu ; a, z) .
$$

Clearly, therefore, Equation (1.23) exhibits the fact that the generalized Hurwitz-Lerch zeta function $\zeta(s, \mu ; a, z)$ of order $\mu$, which was considered recently by Bayad $e t a l$. (see [2], [3] and [7]), is merely a constant multiple of the widely- and extensidely-investigated extended Hurwitz-Lerch zeta function $\Phi_{\mu}^{*}(z, s, a)$ defined by (1.18).

In our present systematic investigation of the $\lambda$-generalized Hurwitz-Lerch zeta function

$$
\Phi_{\lambda_{1}, \cdots, \lambda_{p} ; \mu_{1}, \cdots, \mu_{q}}^{\left(\rho_{1}, \cdots, \rho_{q}, \sigma_{1}, \cdots, \sigma_{q}\right)}(z, s, a ; b, \lambda)
$$

defined by (1.14), we make use also of the widely-studied $H$-function of Charles Fox (1897-1997), which is defined by (see, for details, [21, p. 2, Definition 1.1]; see also [13, p. 1 et seq.], [31, p. 10 et seq.] and [35, p. 49 et seq. ])

$$
\begin{aligned}
H_{p, q}^{m, n}(z) & =H_{p, q}^{m, n}\left[z \mid \begin{array}{l}
\left(a_{p}, A_{p}\right) \\
\left(b_{q}, B_{q}\right)
\end{array}\right] \\
& =H_{p, q}^{m, n}\left[z \mid \begin{array}{l}
\left(a_{1}, A_{1}\right), \cdots,\left(a_{p}, A_{p}\right) \\
\left(b_{1}, B_{1}\right), \cdots,\left(b_{q}, B_{q}\right)
\end{array}\right] \\
& =\frac{1}{2 \pi \mathrm{i}} \int_{\mathscr{L}}^{\Xi(\mathfrak{s}) z^{-\mathfrak{s}} \mathrm{d} \mathfrak{s},}
\end{aligned}
$$

where

$$
\Xi(\mathfrak{s})=\frac{\prod_{j=1}^{m} \Gamma\left(b_{j}+B_{j} \mathfrak{s}\right) \prod_{j=1}^{n} \Gamma\left(1-a_{j}-A_{j} \mathfrak{s}\right)}{\prod_{j=m+1}^{q} \Gamma\left(1-b_{j}-B_{j} \mathfrak{s}\right) \prod_{j=n+1}^{p} \Gamma\left(a_{j}+A_{j} \mathfrak{s}\right)} .
$$

Here

$$
z \in \mathbb{C} \backslash\{0\} \quad \text { with } \quad|\arg (z)|<\pi,
$$

an empty product is interpreted as $1, m, n, p$ and $q$ are integers such that $1 \leqq m \leqq q$ and $0 \leqq n \leqq p$, $A_{j}>0(j=1, \cdots, p) \quad$ and $\quad B_{j}>0(j=1, \cdots, q)$, $\alpha_{j} \in \mathbb{C}(j=1, \cdots, p) \quad$ and $\quad \beta_{j} \in \mathbb{C}(j=1, \cdots, q)$, and $\mathscr{L}$ is a suitable Mellin-Barnes type contour separating the poles of the gamma functions

$$
\left\{\Gamma\left(b_{j}+B_{j} \mathfrak{s}\right)\right\}_{j=1}^{m}
$$

from the poles of the gamma functions

$$
\left\{\Gamma\left(1-a_{j}-A_{j} \mathfrak{s}\right)\right\}_{j=1}^{n} .
$$

The relatively more familiar $G$-function $G_{p, q}^{m, n}(z)$ of Cornelis Simon Meijer (1904-1974) is a special case of Fox's $H$-function defined by (1.24), and we have the following relationship (see, for details, [22, p. 415]; see also [5] and [20]):

$$
G_{p, q}^{m, n}(z)=G_{p, q}^{m, n}\left(z \mid \begin{array}{l}
\left(a_{j}\right)_{j=1}^{p} \\
\left(b_{j}\right)_{j=1}^{q}
\end{array}\right):=H_{p, q}^{m, n}\left[z \mid \begin{array}{l}
\left(a_{1}, 1\right), \cdots,\left(a_{p}, 1\right) \\
\left(b_{1}, 1\right), \cdots,\left(b_{q}, 1\right)
\end{array}\right],
$$

where, for convenience,

$$
G_{p, q}^{m, n}\left(z \mid \begin{array}{l}
\left(a_{j}\right)_{j=1}^{p} \\
\left(b_{j}\right)_{j=1}^{q}
\end{array}\right):=G_{p, q}^{m, n}\left(z \mid \begin{array}{l}
a_{1}, \cdots, a_{p} \\
b_{1}, \cdots, b_{q}
\end{array}\right) .
$$

\section{Explicit Series and Mellin-Barnes Type Contour Integral Representations} below.

Our first set of results are contained in Theorem 1

Theorem 1. The following explicit series and Mellin-Barnes type contour integral representation formulas hold true for the extended Hurwitz-Lerch zeta function

$$
\Phi_{\lambda_{1}, \cdots, \lambda_{p} ; \mu_{1}, \cdots, \mu_{q}}^{\left(\rho_{1}, \cdots, \rho_{q}, \sigma_{1}, \cdots, \sigma_{q}\right)}(z, s, a ; b, \lambda)
$$

defined by (1.14) : 


$$
\begin{aligned}
& \Phi_{\lambda_{1}, \cdots, \lambda_{p} ; \mu_{1}, \cdots, \mu_{q}}^{\left(\rho_{1}, \cdots, \rho_{p}, \sigma_{1}, \cdots, \sigma_{q}\right)}(z, s, a ; b, \lambda) \\
& =\frac{1}{\lambda \Gamma(s)} \sum_{n=0}^{\infty} \frac{\prod_{j=1}^{p}\left(\lambda_{j}\right)_{n \rho_{j}}}{(a+n)^{s} \cdot \prod_{j=1}^{q}\left(\mu_{j}\right)_{n \sigma_{j}}} \\
& \cdot H_{0,2}^{2,0}\left[(a+n) b^{\frac{1}{\lambda}} \mid \frac{z^{n}}{n !}\right.
\end{aligned}
$$

$$
(\lambda>0)
$$

and

$$
\begin{gathered}
\Phi_{\lambda_{1}, \cdots, \lambda_{p} ; \mu_{1}, \cdots, \mu_{q}}^{\left(\rho_{1}, \cdots, \rho_{p}, \sigma_{1}, \cdots, \sigma_{q}\right)}(z, s, a ; b, \lambda)=\frac{\prod_{j=1}^{q} \Gamma\left(\mu_{j}\right)}{2 \pi \mathrm{i} \lambda \Gamma(s) \prod_{j=1}^{p} \Gamma\left(\lambda_{j}\right)} \\
\cdot \int_{-\mathrm{i} \infty}^{\mathrm{i} \infty} \frac{\Gamma(\mathfrak{s}) \Gamma \prod_{j=1}^{p} \Gamma\left(\lambda_{j}-\mathfrak{s} \rho_{j}\right)}{(a-\mathfrak{s})^{s} \cdot \prod_{j=1}^{q} \Gamma\left(\mu_{j}-\mathfrak{s} \sigma_{j}\right)} \\
\cdot H_{0,2}^{2,0}\left[\begin{array}{c}
(a-\mathfrak{s}) b^{\frac{1}{\lambda}} \mid \frac{(s, 1),\left(0, \frac{1}{\lambda}\right)}{(\lambda>0),} \\
(\lambda)^{-\mathfrak{s}} \mathrm{d} \mathfrak{s} \quad(2.2)
\end{array}\right.
\end{gathered}
$$

provided that each member of the assertions (2.1) and (2.2)exists.

Proof. By making use of the series expansion of the Fox-Wright function

$$
{ }_{p} \Psi_{q}^{*}\left[\begin{array}{l}
\left(\lambda_{1}, \rho_{1}\right), \cdots,\left(\lambda_{p}, \rho_{p}\right) ; z e^{-t} \\
\left(\mu_{1}, \sigma_{1}\right), \cdots,\left(\mu_{q}, \sigma_{q}\right) ;
\end{array}\right]
$$

occurring in the integrand of (1.14) and evaluating the resulting integral, in terms of Fox's $H$-function defined by (1.24), by means of the following corrected version of a known integral formula [21, p. 10, Equation (1.53)]:

$$
\begin{aligned}
& \int_{0}^{\infty} t^{a-1} \exp \left(-b t-\frac{c}{t^{\rho}}\right) \mathrm{d} t \\
&= \frac{1}{\rho b^{a}} H_{0,2}^{2,0}\left[b c^{\frac{1}{\rho}} \mid \overline{\left.(a, 1),\left(0, \frac{1}{\rho}\right)\right]}\right] \\
&(\min \{\Re(a), \Re(b), \Re(c)\}>0 ; \rho>0),
\end{aligned}
$$

we obtain the series representation (2.1).

Our demonstration of the Mellin-Barnes type contour integral representation (2.2) is much akin to that of the series representation (2.1). We, therefore, omit the details involved.

In our derivation of each of the representation formulas (2.1) and (2.2), it is assumed that the required inversions of the order of summation and integration are justified by absolute and uniform convergence of the series and integrals involved. The final results (2.1) and (2.2) would thus hold true whenever each member of the assertions (2.1) and (2.2) of Theorem 1 exists.

Remark 2. For the function $\Theta_{\mu}^{\lambda}(z, s, a ; b)$ defined by (1.16), the following special cases of Theorem 1 were derived in [18]:

$$
\begin{gathered}
\Theta_{\mu}^{\lambda}(z, s, a ; b)=\frac{1}{\lambda \Gamma(s)} \sum_{n=0}^{\infty} \frac{(\mu)_{n}}{(a+n)^{s}} \\
\cdot H_{0,2}^{2,0}\left[(a+n) b^{\frac{1}{\lambda}} \mid \overline{(s, 1),\left(0, \frac{1}{\lambda}\right)}\right] \frac{z^{n}}{n !}
\end{gathered}
$$

$$
(\lambda>0)
$$

and

$$
\begin{gathered}
\Theta_{\mu}^{\lambda}(z, s, a ; b)=\frac{1}{2 \pi \mathrm{i} \lambda \Gamma(s) \Gamma(\mu)} \int_{-\mathrm{i} \infty}^{\mathrm{i} \infty} \frac{\Gamma(\mathfrak{s}) \Gamma(\mu-\mathfrak{s})}{(a-\mathfrak{s})^{s}} \\
\cdot H_{0,2}^{2,0}\left[\begin{array}{l|l}
(a-\mathfrak{s}) b^{\frac{1}{\lambda}} \mid- \\
(s, 1),\left(0, \frac{1}{\lambda}\right)
\end{array}\right](-z)^{-\mathfrak{s}} \mathrm{d} \mathfrak{s} \quad(2.5) \\
(\lambda>0),
\end{gathered}
$$

it being assumed that each member of the assertions (2.4) and (2.5) exists (see, for details, [18]).

We now turn toward some series representations and other related results for the extended Hurwitz-Lerch zeta function

$$
\Phi_{\lambda_{1}, \cdots, \lambda_{p} ; \mu_{1}, \cdots, \mu_{q}}^{\left(\rho_{1}, \cdots, \rho_{p}, \sigma_{1}, \cdots, \sigma_{q}\right)}(z, s, a ; b, \lambda)
$$

defined by (1.14). We first give a pair of new series representations involving the the familiar Laguerre polynomials $L_{n}^{(\alpha)}(x)$ of order (index) $\alpha$ and degree $n$ in $x$, defined by

$$
\begin{aligned}
L_{n}^{(\alpha)}(x) & :=\sum_{k=0}^{n}\left(\begin{array}{c}
n+\alpha \\
n-k
\end{array}\right) \frac{(-x)^{k}}{k !} \\
& =\left(\begin{array}{c}
n+\alpha \\
n
\end{array}\right){ }_{1} F_{1}\left[\begin{array}{c}
-n ; \\
\alpha+1 ;
\end{array}\right]
\end{aligned}
$$

in terms of the Kummer's confluent hypergeometric function ${ }_{1} F_{1}$, which are generated by (see, for example, [35, p. 84, Equations 1.11(14)])

$$
(1-t)^{-\alpha-1} \exp \left(-\frac{x t}{1-t}\right)=\sum_{n=0}^{\infty} L_{n}^{(\alpha)}(x) t^{n}
$$




$$
(|t|<1 ; \alpha \in \mathbb{C}) .
$$

Indeed, upon setting

$$
t \rightarrow 1-t^{\lambda} \quad \text { and } \quad x=b
$$

in (2.7), we get

$$
\exp \left(-\frac{b}{t^{\lambda}}\right)=t^{\lambda(\alpha+1)} e^{-b} \sum_{n=0}^{\infty} L_{n}^{(\alpha)}(b)\left(1-t^{\lambda}\right)^{n} .
$$
of

We now make use of (2.8) and the series expansions $\left(1-t^{\lambda}\right)^{n} \quad$ and $\quad{ }_{p} \Psi_{q}^{*}\left[\begin{array}{l}\left(\lambda_{1}, \rho_{1}\right), \cdots,\left(\lambda_{p}, \rho_{p}\right) ; z e^{-t} \\ \left(\mu_{1}, \sigma_{1}\right), \cdots,\left(\mu_{q}, \sigma_{q}\right) ;\end{array}\right]$ occurring in the integrand of (1.14). If we evaluate the resulting Eulerian integral, we are led easily to the series representations given by Theorem 2 below.

Theorem 2. Each of the following series representations holds true for the generalized Hurwitz-Lerch zeta function

$$
\Phi_{\lambda_{1}, \cdots, \lambda_{p} ; \mu_{1}, \cdots, \mu_{q}}^{\left(\rho_{1}, \cdots, \rho_{p}, \sigma_{1}, \cdots, \sigma_{q}\right)}(z, s, a ; b, \lambda)
$$

defined by (1.14) :

$$
\begin{gathered}
\Phi_{\lambda_{1}, \cdots, \lambda_{p} ; \mu_{1}, \cdots, \mu_{q}}^{\left(\rho_{1}, \cdots, \rho_{p}, \sigma_{1}, \cdots, \sigma_{q}\right)}(z, s, a ; b, \lambda) \\
=\frac{e^{-b}}{\Gamma(s)} \sum_{n, \ell=0}^{\infty} \sum_{k=0}^{n}(-1)^{k}\left(\begin{array}{l}
n \\
k
\end{array}\right) \frac{\prod_{j=1}^{p}\left(\lambda_{j}\right) \ell \rho_{j}}{\ell ! \cdot \prod_{j=1}^{q}\left(\mu_{j}\right)_{\ell \sigma_{j}}} \\
\cdot \Gamma(s+\lambda(\alpha+k+1)) L_{n}^{(\alpha)}(b) \frac{z^{\ell}}{(a+\ell)^{s+\lambda(\alpha+k+1)}} \\
(\Re(a)>0 ; \Re(s+\lambda \alpha)>-\lambda)
\end{gathered}
$$

and

$$
\begin{gathered}
\Phi_{\lambda_{1}, \cdots, \lambda_{p} ; \mu_{1}, \cdots, \mu_{q}}^{\left(\rho_{1}, \cdots, \rho_{p}, \sigma_{1}, \cdots, \sigma_{q}\right)}(z, s, a ; b, \lambda) \\
=\frac{e^{-b}}{\Gamma(s)} \sum_{n=0}^{\infty} \sum_{k=0}^{n}(-1)^{k}\left(\begin{array}{l}
n \\
k
\end{array}\right) \Gamma(s+\lambda(\alpha+k+1)) L_{n}^{(\alpha)}(b) \\
\cdot \Phi_{\lambda_{1}, \cdots, \lambda_{p} ; \mu_{1}, \cdots, \mu_{q}}^{\left(\rho_{1}, \cdots, \sigma_{p}, \sigma_{1}, \cdots, \sigma_{q}\right)}(z, s+\lambda(\alpha+j+1), a) \\
(\Re(a)>0 ; \Re(s+\lambda \alpha)>-\lambda)
\end{gathered}
$$

provided that each member of the assertions (2.9) and (2.10) exists,

$$
\Phi_{\lambda_{1}, \cdots, \lambda_{p} ; \mu_{1}, \cdots, \mu_{q}}^{\left(\rho_{1}, \cdots, \rho_{p}, \sigma_{1}, \cdots, \sigma_{q}\right)}(z, s, a)
$$

being given by (1.10).
Proof. As already outlined above, our demonstration of the first assertion (2.9) of Theorem 2 is based essentially upon the representation (2.8) and the following well-known Eulerian integral:

$$
\begin{aligned}
& \int_{0}^{\infty} t^{\rho-1} e^{-\sigma t} \mathrm{~d} t=\frac{\Gamma(\rho)}{\sigma^{\rho}} \\
& (\min \{\Re(\rho), \Re(\sigma)\}>0) .
\end{aligned}
$$

The second assertion (2.13) follows from the first assertion (2.9) when we interpret the $\ell$-series in (2.10) by means of the definition (1.10).

Just as in our demonstration of Theorem 1, it is tacitly assumed that the required inversions of the order of summation and integration are justified by absolute and uniform convergence of the series and integrals involved. The final results (2.9) and (2.10) would thus hold true whenever each member of the assertions (2.9) and (2.10) of Theorem 2 exists.

Remark 3. By suitably specializing Theorem 2, we obtain the following known series representations for the generalized Hurwitz-Lerch zeta function $\Theta_{\mu}^{\lambda}(z, s, a ; b)$ defined by (1.16):

$$
\begin{gathered}
\Theta_{\mu}^{\lambda}(z, s, a ; b)=\frac{e^{-b}}{\Gamma(s)} \sum_{n, \ell=0}^{\infty} \sum_{k=0}^{n}(-1)^{k}\left(\begin{array}{l}
n \\
k
\end{array}\right)\left(\begin{array}{c}
\mu+\ell-1 \\
\ell
\end{array}\right) \\
\cdot \Gamma(s+\lambda(\alpha+k+1)) L_{n}^{(\alpha)}(b) \frac{z^{\ell}}{(a+\ell)^{s+\lambda(\alpha+k+1)}} \\
(\Re(a)>0 ; \Re(s+\lambda \alpha)>-\lambda)
\end{gathered}
$$

and

$$
\begin{gathered}
\Theta_{\mu}^{\lambda}(z, s, a ; b) \\
=\frac{e^{-b}}{\Gamma(s)} \sum_{n=0}^{\infty} \sum_{k=0}^{n}(-1)^{k}\left(\begin{array}{c}
n \\
j
\end{array}\right) \Gamma(s+\lambda(\alpha+k+1)) \\
\cdot L_{n}^{(\alpha)}(b) \Phi_{\mu}^{*}(z, s+\lambda(\alpha+k+1), a) \\
(\Re(a)>0 ; \Re(s+\lambda \alpha)>-\lambda),
\end{gathered}
$$

provided that each member of the assertions (2.12) and (2.13) exists, $\Phi_{\mu}^{*}(z, s, a)$ being given by (1.18) (see, for details, [34]).

Remark 4. For the extended Hurwitz zeta function $\zeta_{b}(s, a)$ defined by (1.20), it is easily deduced from the assertion (2.13) of Theorem 2 when $\lambda=\mu=1$ and $z=1$ that

$$
\begin{gathered}
\zeta_{b}(s, a)=\frac{e^{-b}}{\Gamma(s)} \sum_{n=0}^{\infty} \sum_{j=0}^{n}(-1)^{j}\left(\begin{array}{l}
n \\
j
\end{array}\right) \Gamma(s+\alpha+j+1) \\
\cdot L_{n}^{(\alpha)}(b) \zeta(s+\alpha+j+1, a)
\end{gathered}
$$

$$
(\Re(a)>0 ; \Re(s+\alpha)>-1),
$$


provided that each member of (2.14) exists, $\zeta(s, a)$ being the Hurwitz (or generalized) zeta function given by (1.3). The obvious further special case of (2.14) when $a=1$ and $\alpha=0$ would yield the corrected version of a known result (see [4, p. 298, Equation (7.78)]).

Lastly, we choose give several pairs of summation formulas involving the the generalized Hurwitz-Lerch zeta function

$$
\Phi_{\lambda_{1}, \cdots, \lambda_{p} ; \mu_{1}, \cdots, \mu_{q}}^{\left(\rho_{1}, \cdots, \rho_{p}, \sigma_{1}, \cdots, \sigma_{q}\right)}(z, s, a ; b, \lambda)
$$

defined by (1.14). First of all, it is easily seen from the first assertion (2.1) of Theorem 1 that

$$
\begin{gathered}
\Phi_{\lambda_{1}, \cdots, \lambda_{p} ; \mu_{1}, \cdots, \mu_{q}}^{\left(\rho_{1}, \cdots, \rho_{p}, \sigma_{1}, \cdots, \sigma_{q}\right)}(z, s, a ; b, \lambda) \\
+\Phi_{\lambda_{1}, \cdots, \lambda_{p} ; \mu_{1}, \cdots, \mu_{q}}^{\left(\rho_{1}, \cdots, \rho_{p}, \sigma_{1}, \cdots, \sigma_{q}\right)}(-z, s, a ; b, \lambda) \\
=\frac{2}{\lambda \Gamma(s)} \sum_{n=0}^{\infty} \frac{\prod_{j=1}^{p}\left(\lambda_{j}\right)_{2 n \rho_{j}}}{(a+2 n)^{s} \cdot \prod_{j=1}^{q}\left(\mu_{j}\right)_{2 n \sigma_{j}}} \\
\cdot H_{0,2}^{2,0}\left[\begin{array}{c}
(a+2 n) b^{\frac{1}{\lambda}} \mid \frac{z^{2 n}}{(2 n) !} \\
\left.(s, 1),\left(0, \frac{1}{\lambda}\right)\right] \\
(\lambda>0)
\end{array}\right.
\end{gathered}
$$

and

$$
\begin{aligned}
& \Phi_{\lambda_{1}, \cdots, \lambda_{p} ; \mu_{1}, \cdots, \mu_{q}}^{\left(\rho_{1}, \cdots, \rho_{p}, \sigma_{1}, \cdots, \sigma_{q}\right)}(z, s, a ; b, \lambda) \\
& -\Phi_{\lambda_{1}, \cdots, \lambda_{p} ; \mu_{1}, \cdots, \mu_{q}}^{\left(\rho_{1}, \cdots, \rho_{p}, \sigma_{1}, \cdots, \sigma_{q}\right)}(-z, s, a ; b, \lambda) \\
& =\frac{2}{\lambda \Gamma(s)} \sum_{n=0}^{\infty} \frac{\prod_{j=1}^{p}\left(\lambda_{j}\right)_{(2 n+1) \rho_{j}}}{(a+2 n+1)^{s} \cdot \prod_{j=1}^{q}\left(\mu_{j}\right)_{(2 n+1) \sigma_{j}}} \\
& \cdot H_{0,2}^{2,0}\left[\begin{array}{l|l}
(a+2 n+1) b^{\frac{1}{\lambda}} & \\
& (s, 1),\left(0, \frac{1}{\lambda}\right)
\end{array}\right] \\
& \frac{z^{2 n+1}}{(2 n+1) !} \quad(\lambda>0) \text {. }
\end{aligned}
$$

Alternative expressions for the first members of the summation formulas (2.15) and (2.16) are given by Theorem 3 below.

Theorem 3. Each of the following summation formulas holds true for the the generalized Hurwitz-Lerch zeta function

$$
\Phi_{\lambda_{1}, \cdots, \lambda_{p} ; \mu_{1}, \cdots, \mu_{q}}^{\left(\rho_{1}, \cdots, \rho_{p}, \sigma_{1}, \cdots, \sigma_{q}\right)}(z, s, a ; b, \lambda)
$$

defined by (1.14) :

$$
\begin{aligned}
& 2^{s-1}\left[\Phi_{\lambda_{1}, \cdots, \lambda_{p} ; \mu_{1}, \cdots, \mu_{q}}^{\left(\rho_{1}, \cdots, \rho_{p}, \sigma_{1}, \cdots, \sigma_{q}\right)}(z, s, a ; b, \lambda)\right. \\
& \left.+\Phi_{\lambda_{1}, \cdots, \lambda_{p} ; \mu_{1}, \cdots, \mu_{q}}^{\left(\rho_{1}, \cdots, \rho_{p}, \sigma_{1}, \cdots, \sigma_{q}\right)}(-z, s, a ; b, \lambda)\right] \\
& =\Phi_{\lambda_{1}, \cdots, \lambda_{p}, \frac{1}{2}, \mu_{1}, \cdots, \mu_{q}}^{\left(2 \rho_{1}, \cdots, 2 \rho_{p}, 1,2 \sigma_{1}, \cdots, 2 \sigma_{q}\right)}\left(\frac{z^{2}}{4}, s, \frac{a}{2} ; 2^{\lambda} b, \lambda\right)
\end{aligned}
$$

and

$$
\begin{gathered}
2^{s-1}\left[\Phi_{\lambda_{1}, \cdots, \lambda_{p} ; \mu_{1}, \cdots, \mu_{q}}^{\left(\rho_{1}, \cdots, \rho_{p}, \sigma_{1}, \sigma_{q}\right)}(z, s, a ; b, \lambda)\right. \\
\left.\quad-\Phi_{\lambda_{1}, \cdots, \lambda_{p} ; \mu_{1}, \cdots, \mu_{q}}^{\left(\rho_{1}, \cdots, \rho_{p}, \sigma_{1}, \cdots, \sigma_{q}\right)}(-z, s, a ; b, \lambda)\right] \\
=\frac{\left(\lambda_{1}\right)_{\rho_{1}} \cdots\left(\lambda_{p}\right) \rho_{p}}{\left(\mu_{1}\right)_{\sigma_{1}} \cdots\left(\mu_{q}\right)_{\sigma_{q}}} z \Phi_{\lambda_{1}+\rho_{1}, \cdots, \lambda_{p}+\rho_{p} ; \frac{3}{2}, \mu_{1}+\sigma_{1}, \cdots, \mu_{q}+\sigma_{q}}^{\left(2 \rho_{1}, \cdots, 2 \rho_{p}, 1,2 \sigma_{1}, \cdots, 2 \sigma_{q}\right)} \\
\left(\frac{z^{2}}{4}, s, \frac{a+1}{2} ; 2^{\lambda} b, \lambda\right),
\end{gathered}
$$

provided that each member of the assertions (2.17) and (2.18) exists.

Proof. In view of the definition (1.14), we get

$$
\begin{aligned}
\Phi_{\lambda_{1}, \cdots, \lambda_{p} ; \mu_{1}, \cdots, \mu_{q}}^{\left(\rho_{1}, \cdots, \rho_{p}, \sigma_{1}, \cdots, \sigma_{q}\right)}(z, s, a ; b, \lambda) & \\
& +\Phi_{\lambda_{1}, \cdots, \lambda_{p} ; \mu_{1}, \cdots, \mu_{q}}^{\left(\rho_{1}, \cdots, \rho_{p}, \sigma_{1}, \cdots, \sigma_{q}\right)}(z, s, a ; b, \lambda) \\
= & \frac{1}{\Gamma(s)} \int_{0}^{\infty} t^{s-1} \exp \left(-a t-\frac{b}{t^{\lambda}}\right) \\
& \left.\left(\begin{array}{l}
{ }_{p} \Psi_{q}^{*}\left[\begin{array}{l}
\left(\lambda_{1}, \rho_{1}\right), \cdots,\left(\lambda_{p}, \rho_{p}\right) ; \\
\left(\mu_{1}, \sigma_{1}\right), \cdots,\left(\mu_{q}, \sigma_{q}\right) ;
\end{array}\right] \\
\left.\left[\begin{array}{l}
\left(\lambda_{1}, \rho_{1}\right), \cdots,\left(\lambda_{p}, \rho_{p}\right) ; \\
\left(\mu_{1}, \sigma_{1}\right), \cdots,\left(\mu_{q}, \sigma_{q}\right) ;
\end{array}\right]\right) \mathrm{d} t
\end{array}\right]\right)
\end{aligned}
$$

which readily simplifies to the following form:

$$
\begin{aligned}
& \Phi_{\lambda_{1}, \cdots, \lambda_{p} ; \mu_{1}, \cdots, \mu_{q}}^{\left(\rho_{1}, \cdots, \rho_{p}, \sigma_{1}, \cdots, \sigma_{q}\right)}(z, s, a ; b, \lambda) \\
& \quad+\Phi_{\lambda_{1}, \cdots, \lambda_{p} ; \mu_{1}, \cdots, \mu_{q}}^{\left(\rho_{1}, \cdots, \rho_{p}, \cdots, \sigma_{q}\right)}(z, s ; b, \lambda) \\
& =\frac{2}{\Gamma(s)} \int_{0}^{\infty} t^{s-1} \exp \left(-a t-\frac{b}{t^{\lambda}}\right) \\
& { }_{p} \Psi_{q+1}^{*}\left[\begin{array}{c}
\left(\lambda_{1}, \rho_{1}\right), \cdots,\left(\lambda_{p}, \rho_{p}\right) ; \\
\left(\frac{1}{2}, 1\right),\left(\mu_{1}, \sigma_{1}\right), \cdots,\left(\mu_{q}, \sigma_{q}\right) ;
\end{array} e^{-2 t}\right] \mathrm{d} t .
\end{aligned}
$$

Upon setting

$$
t \rightarrow \frac{t}{2} \quad \text { and } \quad \frac{\mathrm{d} t}{2}
$$


in (2.20), if we interpret the resulting integral by means of the definition (1.14), we arrive at the first assertion (2.17) of Theorem 3. In a similar manner, we can prove the second assertion (2.18) of Theorem 3.

Alternatively, we can derive the assertions (2.17) and (2.18) of Theorem 3 by applying the series representation in (2.1) in order to interpret the second members of (2.15) and (2.16), respectively.

Remark 5. For the particular case $\Theta_{\mu}^{\lambda}(z, s, a ; b)$ defined by (1.16), the following interesting analogues of the assertions (2.17) and (2.18) were derived earlier by Srivastava et al. [34]:

$$
\begin{aligned}
& 2^{s-1}\left[\Theta_{\mu}^{\lambda}(-z, s, a ; b)+\Theta_{\mu}^{\lambda}(z, s, a ; b)\right] \\
& =\sum_{n=0}^{\infty} \frac{(-\mu)_{2 n}}{(2 n) !} \Theta_{\mu}^{\lambda}\left(z^{2}, s, \frac{a}{2}+n ; 2^{\lambda} b\right) z^{2 n}
\end{aligned}
$$

and

$$
\begin{aligned}
2^{s-1} & {\left[\Theta_{\mu}^{\lambda}(-z, s, a ; b)-\Theta_{\mu}^{\lambda}(z, s, a ; b)\right] } \\
= & \sum_{n=0}^{\infty} \frac{(-\mu)_{2 n+1}}{(2 n+1) !} \Theta_{\mu}^{\lambda}\left(z^{2}, s, \frac{a+1}{2}+n ; 2^{\lambda} b\right) z^{2 n+1},
\end{aligned}
$$

provided, of course, that each member of the assertions (2.21) and (2.22) exists. In fact, by putting $\mu=1$ in (2.21) and (2.22), and upon setting $z \rightarrow-z$ and $a \rightarrow 2 a$, Srivastava et al. [34] showed also that

$$
\begin{gathered}
2^{s-1}\left[\Theta_{1}^{\lambda}(z, s, 2 a ; b)+\Theta_{1}^{\lambda}(-z, s, 2 a ; b)\right] \\
=\Theta_{1}^{\lambda}\left(z^{2}, s, a ; 2^{\lambda} b\right)
\end{gathered}
$$

and

$$
\begin{gathered}
2^{s-1}\left[\Theta_{1}^{\lambda}(z, s, 2 a ; b)-\Theta_{1}^{\lambda}(-z, s, 2 a ; b)\right] \\
=z \Theta_{1}^{\lambda}\left(z^{2}, s, a+\frac{1}{2} ; 2^{\lambda} b\right) .
\end{gathered}
$$

In its further special case when $z=\lambda=1$, the summation formula (2.23) can be shown to correspond to known results (see, for example, [4, Theorem 7.9]; see also [4, pp. 308-309]).

\section{Derivative Properties and Associated Partial Differential Equations}

In this section, we aim at showing that the generalized Hurwitz-Lerch zeta function

$$
\Phi_{\lambda_{1}, \cdots, \lambda_{p} ; \mu_{1}, \cdots, \mu_{q}}^{\left(\rho_{1}, \cdots, \rho_{p}, \sigma_{1}, \cdots, \sigma_{q}\right)}(z, s, a ; b, \lambda)
$$

defined by (1.14) satisfies a partial differential equation when the parameter $\lambda$ is given by

$$
\lambda=\frac{1}{m} \quad(m \in \mathbb{N}) .
$$

We first derive the following lemma which will be useful in the demonstration of our main result of this section (Theorem 4 below).

Lemma (Derivative Property). The following derivative formulas hold true:

$$
\begin{aligned}
& \frac{\left(\mu_{1}\right)_{\sigma_{1}} \cdots\left(\mu_{q}\right)_{\sigma_{q}}}{\left(\lambda_{1}\right)_{\rho_{1}} \cdots\left(\lambda_{p}\right)_{\rho_{p}}} \frac{\mathrm{d}}{\mathrm{d} z}\left\{\Phi_{\lambda_{1}, \cdots, \lambda_{p}, \mu_{1}, \cdots, \mu_{q}}^{\left(\rho_{1}, \cdots, \rho_{p}, \sigma_{1}, \cdots, \sigma_{q}\right)}(z, s, a ; b, \lambda)\right\} \\
& \quad=\Phi_{\lambda_{1}+\rho_{1}, \cdots, \lambda_{p}+\rho_{p} ; \mu_{1}+\sigma_{1}, \cdots, \mu_{q}+\sigma_{q}}^{\left(\rho_{1}, \cdots, \rho_{p}, \sigma_{1}, \cdots, \sigma_{q}\right)}(z, s, a+1 ; b, \lambda) \\
& (\lambda>0)
\end{aligned}
$$

and

$$
\begin{aligned}
\frac{\left(\mu_{1}\right)_{\sigma_{1}} \cdots\left(\mu_{q}\right)_{\sigma_{q}}}{\left(\lambda_{1}\right)_{\rho_{1}} \cdots\left(\lambda_{p}\right) \rho_{p}} \frac{\mathrm{d}}{\mathrm{d} z} & \left\{\Phi_{\lambda_{1}, \cdots, \lambda_{p}, \mu_{1}, \cdots, \mu_{q}}^{\left(\rho_{1}, \cdots, \rho_{p}, \sigma_{1}, \cdots, \sigma_{q}\right)}\left(z, s, a ; b^{\frac{1}{m}}, \frac{1}{m}\right)\right\} \\
= & \Phi_{\lambda_{1}+\rho_{1}, \cdots, \lambda_{p}+\rho_{p} ; \mu_{1}+\sigma_{1}, \cdots, \mu_{q}+\sigma_{q}}^{\left(\rho_{1}, \cdots, \rho_{p}, \sigma_{1}, \cdots, \sigma_{q}\right)} \\
& \left(z, s, a+1 ; b^{\frac{1}{m}}, \frac{1}{m}\right) \quad(m \in \mathbb{N})
\end{aligned}
$$

Proof. Our proofs of the derivative formulas (3.1) and (3.2) are simple and direct. For example, by applying the series representation (2.1), it is easily observed that

$$
\begin{aligned}
& \frac{\left(\mu_{1}\right)_{\sigma_{1}} \cdots\left(\mu_{q}\right)_{\sigma_{q}}}{\left(\lambda_{1}\right)_{\rho_{1}} \cdots\left(\lambda_{p}\right)_{\rho_{p}}} \frac{\mathrm{d}}{\mathrm{d} z}\left\{\Phi_{\lambda_{1}, \cdots, \lambda_{p} ; \mu_{1}, \cdots, \mu_{q}}^{\left(\rho_{1}, \cdots, \rho_{p}, \sigma_{1}, \cdots, \sigma_{q}\right)}(z, s, a ; b, \lambda)\right\} \\
& =\frac{\left(\mu_{1}\right)_{\sigma_{1}} \cdots\left(\mu_{q}\right)_{\sigma_{q}}}{\lambda \Gamma(s)\left(\lambda_{1}\right) \rho_{1} \cdots\left(\lambda_{p}\right) \rho_{p}} \sum_{n=1}^{\infty} \frac{\prod_{j=1}^{p}\left(\lambda_{j}\right)_{n \rho_{j}}}{(a+n)^{s} \cdot \prod_{j=1}^{q}\left(\mu_{j}\right)_{n \sigma_{j}}} \\
& \cdot H_{0,2}^{2,0}\left[(a+n) b^{\frac{1}{\lambda}} \mid \begin{array}{l}
(s, 1),\left(0, \frac{1}{\lambda}\right)
\end{array}\right] \frac{z^{n-1}}{(n-1) !} \\
& =\frac{\left(\mu_{1}\right)_{\sigma_{1}} \cdots\left(\mu_{q}\right)_{\sigma_{q}}}{\lambda \Gamma(s)\left(\lambda_{1}\right) \rho_{\rho_{1}} \cdots\left(\lambda_{p}\right)_{\rho_{p}}} \\
& \cdot \sum_{n=0}^{\infty} \frac{\prod_{j=1}^{p}\left(\lambda_{j}\right)_{(n+1) \rho_{j}}}{(a+n+1)^{s} \cdot \prod_{j=1}^{q}\left(\mu_{j}\right)_{(n+1) \sigma_{j}}} \\
& \cdot H_{0,2}^{2,0}\left[(a+n+1) b^{\frac{1}{\lambda}} \mid \begin{array}{l}
(s, 1),\left(0, \frac{1}{\lambda}\right)
\end{array}\right] \frac{z^{n}}{n !}
\end{aligned}
$$




$$
\begin{aligned}
& =\Phi_{\lambda_{1}+\rho_{1}, \cdots, \lambda_{p}+\rho_{p} ; \mu_{1}+\sigma_{1}, \cdots, \mu_{q}+\sigma_{q}}^{\left(\rho_{1} \cdots, \rho_{p}, \sigma_{1} \cdots, \sigma_{q}\right)} \\
& \quad(z, s, a+1 ; b, \lambda) \quad(\lambda>0),
\end{aligned}
$$

which yields precisely the first assertion (3.1) of the Lemma. The second assertion (3.2) follows immediately from (3.1) upon setting

$$
\lambda=\frac{1}{m} \quad(m \in \mathbb{N}) \quad \text { and } \quad b \rightarrow b^{\frac{1}{m}} \quad(m \in \mathbb{N}) .
$$

Our main result in this section is contained in the following theorem.

Theorem 4. Let $m \in \mathbb{N}$. Then the generalized Hurwitz-Lerch zeta function

$$
\Phi_{\lambda_{1}, \cdots, \lambda_{p}, \mu_{1}, \cdots, \mu_{q}}^{\left(\rho_{1}, \cdots, \rho_{p}, \sigma_{1}, \cdots, \sigma_{q}\right)}\left(z, s, a ; b, \frac{1}{m}\right)
$$

satisfies the following partial differential equation:

$$
\begin{aligned}
& {\left[(-1)^{m+1} m^{m} \mathfrak{D}_{\mathfrak{b}}-(a+1) b^{m} \theta_{z}\right]} \\
& \quad\left\{\Phi_{\lambda_{1}, \cdots, \lambda_{p} ; \mu_{1}, \cdots, \mu_{q}}^{\left(\rho_{1}, \cdots, \rho_{p}, \sigma_{1}, \cdots, \sigma_{q}\right)}\left(z, s, a ; b, \frac{1}{m}\right)\right\}=0,
\end{aligned}
$$

where the differential operators $\mathfrak{D}_{\mathfrak{b}}, \theta_{z}$ and $\theta_{b}$ are given by

$$
\begin{gathered}
\mathfrak{D}_{\mathfrak{b}}:=\theta_{b}\left(\theta_{b}-s\right)\left(\theta_{b}-\frac{1}{m}\right) \cdots\left(\theta_{b}-\frac{m-1}{m}\right), \\
\theta_{z}:=z \frac{\partial}{\partial z} \quad \text { and } \quad \theta_{b}:=b \frac{\partial}{\partial b},
\end{gathered}
$$

respectively.

Proof. First of all, let us rewrite the $H$-function occurring in the Mellin-Barnes type contour integral representation (2.2) as follows:

$$
\begin{aligned}
& H_{0,2}^{2,0}\left[(a-\mathfrak{s}) b^{\frac{1}{\lambda}} \mid \overline{\left.(s, 1),\left(0, \frac{1}{\lambda}\right)\right]}\right. \\
& =\frac{1}{2 \pi \mathrm{i}} \int_{\mathfrak{L}} \Gamma(s+w) \Gamma\left(\frac{w}{\lambda}\right)\left[(a-\mathfrak{s}) b^{\frac{1}{\lambda}}\right]^{-w} \mathrm{~d} w
\end{aligned}
$$

where $\mathfrak{L}$ is a suitable Mellin-Barnes type contour integral in the complex $w$-plane. We now set

$$
\frac{1}{\lambda}=m \quad(m \in \mathbb{N}) \quad \text { and } \quad b \rightarrow b^{\frac{1}{m}} \quad(m \in \mathbb{N})
$$

in the above equation (3.6) and then apply the following well-known (Gauss-Legendre) multiplication formula (see, for example, [1, p. 256, Entry (6.1.18)]):

$$
\Gamma(m z)=(2 \pi)^{\frac{1-m}{2}} m^{m z-\frac{1}{2}} \prod_{j=1}^{m} \Gamma\left(z+\frac{j-1}{m}\right)
$$

$$
\left(z \neq 0,-\frac{1}{m},-\frac{2}{m}, \cdots ; m \in \mathbb{N}\right) .
$$

We thus find that

$$
\begin{aligned}
& H_{0,2}^{2,0}\left[\begin{array}{l|l}
(a-\mathfrak{s}) b & \\
(s, 1),(0, m)
\end{array}\right] \\
& =\frac{1}{2 \pi \mathrm{i}} \int_{\mathfrak{L}} \Gamma(s+w) \Gamma(m w)[(a-\mathfrak{s}) b]^{-w} \mathrm{~d} w \\
& =\frac{(2 \pi)^{\frac{1-m}{2}}}{2 \pi \mathrm{i} \sqrt{m}} \int_{\mathfrak{L}} \Gamma(s+w) \\
& \cdot \prod_{j=1}^{m} \Gamma\left(w+\frac{j-1}{m}\right)\left[(a-\mathfrak{s}) b m^{-m}\right]^{-w} \mathrm{~d} w \\
& =\frac{(2 \pi)^{\frac{1-m}{2}}}{\sqrt{m}} G_{0, m+1}^{m+1,0} \\
& \left(\begin{array}{l|l}
(a-\mathfrak{s}) b m^{-m} & \begin{array}{l}
s, 0, \frac{1}{m}, \frac{2}{m}, \cdots, \frac{m-1}{m}
\end{array}
\end{array}\right),
\end{aligned}
$$

where

$$
G_{0, m+1}^{m+1,0}\left((a-\mathfrak{s}) b m^{-m}\right)
$$

is a very specialized case of Meijer's $G$-function $G_{p, q}^{m, n}(z)$ defined by (1.26).

We know that the function $W$ defined by

$$
W:=G_{p, q}^{m, n}\left(z \mid \begin{array}{l}
a_{1}, \cdots, a_{p} \\
b_{1}, \cdots, b_{q}
\end{array}\right)
$$

satisfies the following differential equation of order $\max (p, q)$ (see, for example, [5, p. 210, Equation 5.4(1)]):

$\left[(-1)^{p-m-n} z\left(\vartheta_{z}-a_{1}+1\right) \cdots\left(\vartheta_{z}-a_{p}+1\right)-\left(\vartheta_{z}-b_{1}\right) \cdots\left(\vartheta_{z}-b_{q}\right)\right] W=0$,

where

$$
\vartheta_{z}=z \frac{\mathrm{d}}{\mathrm{d} z}
$$

Clearly, therefore, the function given by (3.8) satisfies the following differential equation:

$$
\begin{aligned}
& {\left[(-1)^{m+1}(a-\mathfrak{s}) m^{-m} b-\theta_{b}\left(\theta_{b}-s\right)\left(\theta_{b}-\frac{1}{m}\right) \cdots\left(\theta_{b}-\frac{m-1}{m}\right)\right]} \\
& \quad\left\{G_{0, m+1}^{m+1,0}\left((a-\mathfrak{s}) b m^{-m} \mid \frac{\mid}{s, 0, \frac{1}{m}, \frac{2}{m}, \cdots, \frac{m-1}{m}}\right)\right\}=0, \quad \text { (3.10) }
\end{aligned}
$$

where, as already stated in (3.5),

$$
\theta_{b}=b \frac{\partial}{\partial b}
$$

Now, if we write [see also Equation (3.4)]

$$
\mathfrak{D}_{\mathfrak{b}}:=\theta_{b}\left(\theta_{b}-s\right)\left(\theta_{b}-\frac{1}{m}\right) \cdots\left(\theta_{b}-\frac{m-1}{m}\right)\left(\theta_{b}:=b \frac{\partial}{\partial b}\right),
$$


then the equation (3.10) becomes

$$
\begin{aligned}
\mathfrak{D}_{\mathfrak{b}} & \left\{G_{0, m+1}^{m+1,0}\left((a-\mathfrak{s}) b m^{-m} \mid \begin{array}{l}
s, 0, \frac{1}{m}, \frac{2}{m}, \cdots, \frac{m-1}{m}
\end{array}\right)\right\} \\
= & (-1)^{m+1} m^{-m}(a-\mathfrak{s}) b G_{0, m+1}^{m+1,0} \\
& \left(\begin{array}{ll}
(a-\mathfrak{s}) b m^{-m} \mid & s, 0, \frac{1}{m}, \frac{2}{m}, \cdots, \frac{m-1}{m}
\end{array}\right) .
\end{aligned}
$$

By applying the differential operator $\mathfrak{D}_{\mathfrak{b}}$ to the function

$$
\Phi_{\lambda_{1}, \cdots, \lambda_{p} ; \mu_{1}, \cdots, \mu_{q}}^{\left(\rho_{1}, \cdots, \rho_{p}, \sigma_{1}, \cdots, \sigma_{q}\right)}\left(-z, s, a ; b^{\frac{1}{m}}, \frac{1}{m}\right)
$$

given by (2.2) with

$$
z \rightarrow-z, \quad \lambda=\frac{1}{m} \quad(m \in \mathbb{N})
$$

and

$$
b \rightarrow b^{\frac{1}{m}} \quad(m \in \mathbb{N}),
$$

we find by making use of (3.11) that

$$
\begin{aligned}
& \mathfrak{D}_{\mathfrak{b}}\left\{\Phi_{\lambda_{1}, \cdots, \lambda_{p} ; \mu_{1}, \cdots, \mu_{q}}^{\left(\rho_{1}, \cdots, \rho_{1}, \sigma_{1}, \cdots, \sigma_{q}\right)}\left(-z, s, a ; b^{\frac{1}{m}}, \frac{1}{m}\right)\right\} \\
& =\frac{\sqrt{m}(2 \pi)^{\frac{1-m}{2}} \prod_{j=1}^{q} \Gamma\left(\mu_{j}\right)}{2 \pi \mathrm{i} \Gamma(s) \prod_{j=1}^{p} \Gamma\left(\lambda_{j}\right)} \int_{-\mathrm{i} \infty}^{\mathrm{i} \infty} \frac{\Gamma(\mathfrak{s}) \Gamma \prod_{j=1}^{p} \Gamma\left(\lambda_{j}-\mathfrak{s} \rho_{j}\right)}{(a-\mathfrak{s})^{s} \prod_{j=1}^{q} \Gamma\left(\mu_{j}-\mathfrak{s} \sigma_{j}\right)}
\end{aligned}
$$

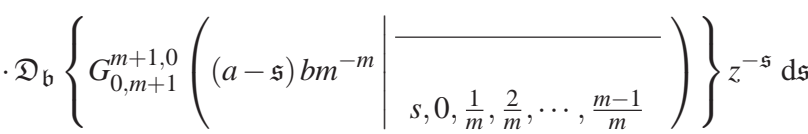

$$
\begin{aligned}
& =\frac{(-1)^{m+1} m^{\frac{1}{2}-m}(2 \pi)^{\frac{1-m}{2}} b \prod_{j=1}^{q} \Gamma\left(\mu_{j}\right)}{2 \pi \mathrm{i} \Gamma(s) \prod_{j=1}^{p} \Gamma\left(\lambda_{j}\right)} \\
& \int_{-\mathrm{i} \infty}^{\mathrm{i} \infty} \frac{\Gamma(\mathfrak{s}) \prod_{j=1}^{p} \Gamma\left(\lambda_{j}-\mathfrak{s} \rho_{j}\right)}{(a-\mathfrak{s})^{s} \prod_{j=1}^{q} \Gamma\left(\mu_{j}-\mathfrak{s} \sigma_{j}\right)} \cdot(a-\mathfrak{s}) \\
& \cdot G_{0, m+1}^{m+1,0}\left(\begin{array}{l|l}
(a-\mathfrak{s}) b m^{-m} & \\
s, 0, \frac{1}{m}, \frac{2}{m}, \cdots, \frac{m-1}{m}
\end{array}\right) z^{-\mathfrak{s} \mathrm{d} \mathfrak{s}} \\
& =:(-1)^{m+1} m^{-m} b\left(a \mathscr{I}_{1}-\mathscr{I}_{2}\right) \text {, }
\end{aligned}
$$

where the first integral $\mathscr{I}_{1}$ is actually the generalized Hurwitz-Lerch zeta function given by

$$
\mathscr{I}_{1}=\Phi_{\lambda_{1}, \cdots, \lambda_{p} ; \mu_{1}, \cdots, \mu_{q}}^{\left(\rho_{1}, \cdots, \rho_{p}, \sigma_{1}, \cdots, \sigma_{q}\right)}\left(-z, s, a ; b^{\frac{1}{m}}, \frac{1}{m}\right) \text {. }
$$

The evaluation of the second integral $\mathscr{I}_{2}$ given by

$$
\mathscr{I}_{2}:=\frac{\sqrt{m}(2 \pi)^{\frac{1-m}{2}} \prod_{j=1}^{q} \Gamma\left(\mu_{j}\right)}{2 \pi \mathrm{i} \Gamma(s) \prod_{j=1}^{p} \Gamma\left(\lambda_{j}\right)}
$$

$$
\begin{aligned}
& \int_{-\mathrm{i} \infty}^{\mathrm{i} \infty} \frac{\Gamma(\mathfrak{s}+1) \prod_{j=1}^{p} \Gamma\left(\lambda_{j}-\mathfrak{s} \rho_{j}\right)}{(a-\mathfrak{s})^{s} \prod_{j=1}^{q} \Gamma\left(\mu_{j}-\mathfrak{s} \sigma_{j}\right)} \\
& \cdot G_{0, m+1}^{m+1,0}\left((a-\mathfrak{s}) b m^{-m} \mid \overline{\left.s, 0, \frac{1}{m}, \frac{2}{m}, \cdots, \frac{m-1}{m}\right) z^{-\mathfrak{s}} \mathrm{d} \mathfrak{s}}\right.
\end{aligned}
$$

is more complicated. Since the residues of $\Gamma(\mathfrak{s}+1)$ at the poles $\mathfrak{s}=-k(k \in \mathbb{N})$ are computed by

$$
\begin{aligned}
\operatorname{Res}_{\mathfrak{s}=-k}\{\Gamma(\mathfrak{s}+1)\} & =\lim _{\mathfrak{s} \rightarrow-k}(\mathfrak{s}+k) \Gamma(\mathfrak{s}+1) \\
& =\frac{(-1)^{k-1}}{(k-1) !}
\end{aligned}
$$

the Residue Theorem implies that

$$
\begin{aligned}
& \mathscr{I}_{2}=\frac{\sqrt{m}(2 \pi)^{\frac{1-m}{2}} \prod_{j=1}^{q} \Gamma\left(\mu_{j}\right)}{\Gamma(s) \prod_{j=1}^{p} \Gamma\left(\lambda_{j}\right)} \\
& \cdot \sum_{k=1}^{\infty} \frac{\prod_{j=1}^{p} \Gamma\left(\lambda_{j}+k \rho_{j}\right)}{(a+k)^{s} \prod_{j=1}^{q} \Gamma\left(\mu_{j}+k \sigma_{j}\right)} z^{k} \operatorname{Res}_{\mathfrak{s}=-k}\{\Gamma(\mathfrak{s}+1)\} \\
& \cdot G_{0, m+1}^{m+1,0}\left(\begin{array}{l|l}
(a+k) b m^{-m} & \begin{array}{l}
s, 0, \frac{1}{m}, \frac{2}{m}, \cdots, \frac{m-1}{m}
\end{array}
\end{array}\right. \\
& =\frac{\sqrt{m}(2 \pi)^{\frac{1-m}{2}} \prod_{j=1}^{q} \Gamma\left(\mu_{j}\right)}{\Gamma(s) \prod_{j=1}^{p} \Gamma\left(\lambda_{j}\right)} \\
& \cdot \sum_{k=1}^{\infty} \frac{\prod_{j=1}^{p} \Gamma\left(\lambda_{j}+k \rho_{j}\right)}{(a+k)^{s} \prod_{j=1}^{q} \Gamma\left(\mu_{j}+k \sigma_{j}\right)} \frac{(-1)^{k-1} z^{k}}{(k-1) !} \\
& G_{0, m+1}^{m+1,0}\left(\begin{array}{l|l}
(a+k) b m^{-m} & \\
s, 0, \frac{1}{m}, \frac{2}{m}, \cdots, \frac{m-1}{m}
\end{array}\right) \\
& =\frac{z \sqrt{m}(2 \pi)^{\frac{1-m}{2}} \prod_{j=1}^{q} \Gamma\left(\mu_{j}\right)}{\Gamma(s) \prod_{j=1}^{p} \Gamma\left(\lambda_{j}\right)} \\
& \cdot \sum_{k=0}^{\infty} \frac{\prod_{j=1}^{p} \Gamma\left(\lambda_{j}+(k+1) \rho_{j}\right)}{(a+1+k)^{s} \prod_{j=1}^{q} \Gamma\left(\mu_{j}+(k+1) \rho_{j}\right)} \frac{(-z)^{k}}{k !} \\
& \cdot G_{0, m+1}^{m+1,0}\left(\begin{array}{l|l}
(a+1+k) b m^{-m} & \\
s, 0, \frac{1}{m}, \frac{2}{m}, \cdots, \frac{m-1}{m}
\end{array}\right) \\
& =\frac{m z \lambda_{1} \cdots \lambda_{p}}{\mu_{1} \cdots \mu_{q} \Gamma(s)}
\end{aligned}
$$




$$
\begin{gathered}
\cdot \sum_{k=0}^{\infty} \frac{\prod_{j=1}^{p} \Gamma\left(\lambda_{j}+1\right)_{k \lambda_{j}}}{(a+1+k)^{s} \prod_{j=1}^{q} \Gamma\left(\mu_{j}+1\right)_{k \mu_{j}}} \frac{(-z)^{k}}{k !} \\
\cdot H_{0,2}^{2,0}[(a+1+k) b \mid \overline{(s, 1),(0, m)}] \\
\frac{z \lambda_{1} \cdots \lambda_{p}}{\mu_{1} \cdots \mu_{q}} \\
\cdot \Phi_{\lambda_{1}+1, \cdots, \lambda_{p}+1 ; \mu_{1}+1, \cdots, \mu_{q}+1}^{\left(\rho_{1}, \cdots, \rho_{p}, \sigma_{1} \cdots, \sigma_{q}\right)}\left(-z, s, a ; b^{\frac{1}{m}}, \frac{1}{m}\right)
\end{gathered}
$$

Thus, by applying the derivative formula (3.2) in (3.16), we get

$$
\mathscr{I}_{2}=-z \frac{\mathrm{d}}{\mathrm{d} z}\left\{\Phi_{\lambda_{1}, \cdots, \lambda_{p} ; \mu_{1}, \cdots, \mu_{q}}^{\left(\rho_{1}, \cdots, \rho_{p}, \sigma_{1}, \cdots, \sigma_{q}\right)}\left(-z, s, a ; b^{\frac{1}{m}}, \frac{1}{m}\right)\right\} .
$$

Now, upon substituting from (3.13) and (3.17) into (3.12), we obtain

$$
\begin{aligned}
& \mathfrak{D}_{\mathfrak{b}}\left\{\Phi_{\lambda_{1}, \cdots, \lambda_{p} ; \mu_{1}, \cdots, \mu_{q}}^{\left(\rho_{1}, \cdots, \rho_{p}, \sigma_{1}, \cdots, \sigma_{q}\right)}\left(-z, s, a ; b^{\frac{1}{m}}, \frac{1}{m}\right)\right\} \\
& =(-1)^{m+1} m^{-m} a b \Phi_{\lambda_{1}, \cdots, \lambda_{p} ; \mu_{1}, \cdots, \mu_{q}}^{\left(\rho_{1}, \cdots, \rho_{p}, \sigma_{1}, \cdots, \sigma_{q}\right)}\left(-z, s, a ; b^{\frac{1}{m}}, \frac{1}{m}\right) \\
& +(-1)^{m+1} m^{-m} b z \frac{\partial}{\partial z} \\
& { }^{-} \quad\left\{\Phi_{\lambda_{1}, \cdots, \lambda_{p} ; \mu_{1}, \cdots, \mu_{q}}^{\left(\rho_{1}, \cdots, \rho_{p}, \sigma_{1}, \cdots, \sigma_{q}\right)}\left(-z, s, a ; b^{\frac{1}{m}}, \frac{1}{m}\right)\right\},
\end{aligned}
$$

which, after a straightforward simplification, assumes the following form:

$$
\begin{gathered}
{\left[(-1)^{m+1} m^{m} \mathfrak{D}_{\mathfrak{b}}-a b-b \theta_{z}\right]} \\
\left\{\Phi_{\lambda_{1}, \cdots, \lambda_{p}, \mu_{1}, \cdots, \mu_{q}}^{\left(\rho_{1}, \cdots, \rho_{p}, \sigma_{1}, \cdots, \sigma_{q}\right)}\left(-z, s, a ; b^{\frac{1}{m}}, \frac{1}{m}\right)\right\}=0 \\
\left(\theta_{z}:=z \frac{\partial}{\partial z}\right)
\end{gathered}
$$

Finally, by setting

$$
b \rightarrow b^{m} \quad(m \in \mathbb{N}) \quad \text { and } \quad z \rightarrow-z
$$

in the last equation (3.19), we readily arrive at the desired result (3.3) asserted by Theorem 4.

Remark 6. An interesting special case of Theorem 4 occurs when we set $m=1$. We are thus led immediately to the following results.

Theorem 5. The generalized Hurwitz-Lerch zeta function

$$
\Phi_{\lambda_{1}, \cdots, \lambda_{p} ; \mu_{1}, \cdots, \mu_{q}}^{\left(\rho_{1}, \cdots, \rho_{p}, \sigma_{1}, \cdots, \sigma_{q}\right)}(z, s, a ; b, 1)
$$

satisfies the following partial differential equation:

$$
\left[b \frac{\partial}{\partial b}\left(b \frac{\partial}{\partial b}-s\right)\left(b \frac{\partial}{\partial b}-1\right)-(a+1) b z \frac{\partial}{\partial z}\right]
$$

$$
\left\{\Phi_{\lambda_{1}, \cdots, \lambda_{p} ; \mu_{1}, \cdots, \mu_{q}}^{\left(\rho_{1}, \cdots, \rho_{q}, \sigma_{1}, \cdots, \sigma\right)}(z, s, a ; b, 1)\right\}=0
$$

Furthermore, the generalized Hurwitz-Lerch zeta function

$$
\Phi_{\lambda_{1}, \cdots, \lambda_{p} ; \mu_{1}, \cdots, \mu_{q}}^{\left(\rho_{1}, \cdots, \rho_{p}, \sigma_{1}, \cdots, \sigma_{q}\right)}(z, s, a ; b, 1),
$$

when considered as an analytic function of the variable $b$, satisfies the following relationship:

$$
\begin{gathered}
{\left[b \frac{\partial}{\partial b}\left(b \frac{\partial}{\partial b}-s\right)\left(b \frac{\partial}{\partial b}-1\right)\right]} \\
\left\{\Phi_{\lambda_{1}, \cdots, \lambda_{p} ; \mu_{1}, \cdots, \mu_{q}}^{\left(\rho_{1}, \cdots, \rho_{p}, \sigma_{1}, \cdots, \sigma_{q}\right)}(z, s, a ; b, 1)\right\} \\
=\frac{(a+1) b \lambda_{1} \cdots \lambda_{p}}{\mu_{1} \cdots \mu_{q}} \\
\cdot \Phi_{\lambda_{1}, \cdots, \lambda_{p}, \mu_{1}, \cdots, \mu_{q}}^{\left(\rho_{1}, \cdots, \rho_{p}, \sigma_{1}, \cdots, \sigma_{q}\right)}(z, s, a+1 ; b, 1) .
\end{gathered}
$$

\section{Applications Involving the Hurwitz Measure and Probability Distributions}

Let $\chi_{A}(n)$ be the characteristic function of the subset $A$ of the set $\mathbb{N}$ of positive integers (or, in the language of probability theory, the indicator function of the event $A \subseteq$ $\mathbb{N}$ ). Then it is well known that the following arithmetic density of number theory:

$$
\operatorname{dens}(A)=\lim _{k \rightarrow \infty} \frac{1}{k} \sum_{n=1}^{k} \chi_{A}(n)
$$

does not define a measure on the set $\mathbb{N}$ of positive integers. In order to remedy this deficiency, Golomb [11] defined a probability on the sample space $\mathbb{N}$ and showed that, if the subset $A$ of $\mathbb{N}$ has an arithmetic density, then

$$
\lim _{s \rightarrow 1} Q_{s}(A)=\operatorname{dens}(A)
$$

thereby allowing number-theoretic facts regarding densities of sets of positive integers to be proven by probabilistic means and then showing that such properties are preserved in the limit. Subsequently, in an interesting sequel to Golomb's investigation [11], Lippert [17] gave an analogous definition of the probabilities $P_{S}$ when the set $\mathbb{N}$ is replaced by the set of all real numbers greater than 1 . Thus, for a Borel set $A \subseteq(1, \infty)$, Lippert's Hurwitz measure of the set $A$ is defined by (see, for details, [17, p. 279, Definition 1]

$$
P_{S}(A)=\frac{s}{\zeta(s)} \int_{1}^{\infty} \chi_{A}(a) \zeta(s+1, x) \mathrm{d} x
$$

or, equivalently, by

$$
P_{s}(A)=\int_{x \in(1, \infty)} \chi_{A}(x) \mathrm{d} \tilde{\mu}(x, s),
$$


where, in terms of the Hurwitz (or generalized) zeta function $\zeta(s, a)$ defined by (1.3), we have

$$
\tilde{\mu}(x, s):=-\frac{\zeta(s, x)}{\zeta(s)}
$$

and

$$
\mathrm{d} \tilde{\mu}(x, s)=-\frac{\mathrm{d} \zeta(s, x)}{\zeta(s)}=s \frac{\zeta(s+1, x)}{\zeta(s)} \mathrm{d} x
$$

More recently, Srivastava et al. [34] introduced and investigated a new continuous analogue of Lippert's Hurwitz measure in (4.3) by using a special case of the generalized Hurwitz-Lerch zeta function $\Theta_{\mu}^{\lambda}(z, s, a ; b)$ defined by (1.16), that is,

$$
\begin{aligned}
& \Theta_{1}^{\lambda}(1, s, a ; b)=\frac{1}{\Gamma(s)} \int_{0}^{\infty} \frac{t^{s-1}}{1-e^{-t}} \exp \left(-a t-\frac{b}{t^{\lambda}}\right) \mathrm{d} t \\
& =\left.\Phi_{\lambda_{1}, \cdots, \lambda_{p} ; \mu_{1}, \cdots, \mu_{q}}^{\left(\rho_{1}, \cdots, \rho_{p}, \cdots, \sigma_{q}\right)}(1, s, a ; b, \lambda)\right|_{p-1=q=0 \quad\left(\lambda_{1}=\mu=1 ; \rho_{1}=1\right)}
\end{aligned}
$$

Definition 2. A Borel set [named after Émile Borel (18711956)] is any set in a topological space that can be formed from open sets (or, equivalently, from closed sets) through the operations of countable union, countable intersection and relative complement. Thus, for a Borel set $A \subseteq(1, \infty)$, the generalized Hurwitz measure of the set $A$ is defined by

$$
\begin{aligned}
P_{S}(A)= & \frac{s}{\Theta_{1}^{\lambda}(1, s, 1 ; b)} \\
& \cdot \int_{1}^{\infty} \chi_{A}(a) \Theta_{1}^{\lambda}(1, s+1, a ; b) \mathrm{d} a
\end{aligned}
$$

or, equivalently, by

$$
P_{s}(A)=\int_{a \in(1, \infty)} \chi_{A}(a) \mathrm{d} \tilde{\mu}(a, s ; b, \lambda)
$$

where

$$
\tilde{\mu}(a, s ; b, \lambda):=-\frac{\Theta_{1}^{\lambda}(1, s, a ; b)}{\Theta_{1}^{\lambda}(1, s, 1 ; b)}
$$

and

$$
\begin{aligned}
\mathrm{d} \tilde{\mu}(a, s ; b, \lambda) & \\
& =-\frac{\mathrm{d} \Theta_{1}^{\lambda}(1, s, a ; b)}{\Theta_{1}^{\lambda}(1, s, 1 ; b)} \\
& =\frac{s}{\Theta_{1}^{\lambda}(1, s, 1 ; b)} \Theta_{1}^{\lambda}(1, s+1, a ; b) \mathrm{d} a,
\end{aligned}
$$

since it is easily seen from the definition (1.16) that

$$
\frac{\mathrm{d}}{\mathrm{d} a}\left\{\Theta_{\mu}^{\lambda}(z, s, a ; b)\right\}=-s \Theta_{\mu}^{\lambda}(z, s+1, a ; b) .
$$

In view of the following relationship:

$$
\begin{aligned}
P_{s}((1, \infty)) & =\int_{1}^{\infty} \mathrm{d} \tilde{\mu}(a, s ; b, \lambda) \\
& =\lim _{a \rightarrow \infty} \tilde{\mu}(a, s ; b, \lambda)-\tilde{\mu}(1, s ; b, \lambda)=1,
\end{aligned}
$$

the generalized Hurwitz measure $P_{S}(A)$ in (4.7) or (4.8) also defines a probability measure on $(1, \infty)$.

Remark 7. For $\lambda=1$ and by letting $b \rightarrow 0$, we have

$$
\lim _{b \rightarrow 0} H_{0,2}^{2,0}\left[a b \mid \begin{array}{l}
(s, 1),(0,1)
\end{array}\right]=\Gamma(s),
$$

which implies that

$$
\begin{aligned}
\lim _{b \rightarrow 0} \tilde{\mu}(a, s ; b, 1) & =-\lim _{b \rightarrow 0} \frac{\Theta_{1}^{1}(1, s, a, b)}{\Theta_{1}^{1}(1, s, 1, b)} \\
& =-\frac{\zeta(s, x)}{\zeta(s)}=: \tilde{\mu}(x, s) .
\end{aligned}
$$

Thus, clearly, $\tilde{\mu}(x, s)$ can be continuously approximated by $\tilde{\mu}(a, s ; b, 1)$.

Theorem 6. The measure $\tilde{\mu}(a, s ; b, \lambda)$ satisfies the following difference equation:

$$
\begin{aligned}
\tilde{\mu}(a+1, s ; b, \lambda)-\tilde{\mu}(a, s ; b, \lambda) \\
=\frac{H_{0,2}^{2,0}\left[a b^{\frac{1}{\lambda}} \mid \overline{(s, 1),\left(0, \frac{1}{\lambda}\right)}\right]}{\lambda a^{s} \Gamma(s) \Theta_{1}^{\lambda}(1, s, 1 ; b)} \\
(s>1 ; a>0 ; b>0 ; \lambda>0) .
\end{aligned}
$$

Proof. From the series representation (2.4) of

$$
\Theta_{\mu}^{\lambda}(z, s, a+1 ; b) \quad(\text { with } \quad \mu=1 \quad \text { and } \quad z=1),
$$

we have

$$
\begin{aligned}
\Theta_{1}^{\lambda}(1, s, a+1 ; b) & \frac{1}{\lambda \Gamma(s)} \sum_{n=0}^{\infty} \frac{1}{(a+n+1)^{s}} \\
& \cdot H_{0,2}^{2,0}\left[(a+n+1) b^{\frac{1}{\lambda}} \mid \overline{\left.(s, 1),\left(0, \frac{1}{\lambda}\right)\right]}\right. \\
= & \frac{1}{\lambda \Gamma(s)} \sum_{n=1}^{\infty} \frac{1}{(a+n)^{s}} \mid \overline{\left.(s, 1),\left(0, \frac{1}{\lambda}\right)\right]} \\
= & \Theta_{1}^{\lambda}(1, s, a ; b)-\frac{1}{\lambda a^{s} \Gamma(s)}
\end{aligned}
$$




$$
\cdot H_{0,2}^{2,0}\left[a b^{\frac{1}{\lambda}} \mid \begin{array}{l}
(s, 1),\left(0, \frac{1}{\lambda}\right)
\end{array}\right] .
$$

The difference equation (4.14) now follows on combining (4.9) and (4.15).

Remark 8. For $\lambda=1$ and by letting $b \rightarrow 0$, the difference equation (4.14) reduces to the following form:

$$
\tilde{\mu}(a+1, s)-\tilde{\mu}(a, s)=\frac{1}{a^{s} \zeta(s)},
$$

where $\tilde{\mu}(x, s)$ is given by (4.5).

For open events, the generalized Hurwitz measure $P_{S}(A)$ in (4.7) or (4.8) can be evaluated by using (4.8) and the above Proposition. The results are being stated as Theorem 7 below.

Theorem 7. If $A=(a, a+1)$, then

$$
\begin{aligned}
P_{s}(A)= & P_{s}((a, a+1)) \\
& H_{0,2}^{2,0}\left[a b^{\frac{1}{\lambda} \mid} \mid \overline{(s, 1),\left(0, \frac{1}{\lambda}\right)}\right] \\
= & \frac{\lambda a^{s} \Gamma(s) \Theta_{1}^{\lambda}(1, s, 1 ; b)}{} .
\end{aligned}
$$

More generally, the generalized Hurwitz measure of an open set $A \subseteq(1, \infty)$ is given by

$$
\begin{aligned}
P_{S}(A) & =\sum_{i \in I} P_{S}\left(\left(a_{i}, b_{i}\right)\right) \\
& =\sum_{i \in I}\left(\frac{\Theta_{1}^{\lambda}\left(1, s, a_{i} ; b\right)-\Theta_{1}^{\lambda}\left(1, s, b_{i} ; b\right)}{\Theta_{1}^{\lambda}(1, s, 1 ; b)}\right),
\end{aligned}
$$

where

$$
A=\bigcup_{i \in I}\left(a_{i}, b_{i}\right) \quad\left(a_{i}, b_{i} \in[1, \infty) ; i \in I\right) .
$$

The following theorem shows that the generalized Hurwitz measure $P_{S}(A)$ in (4.7) or (4.8) basically inherits all properties of Lippert's Hurwitz measure given by (4.3) or (4.4).

Theorem 8. Corresponding to the generalized Hurwitz measure given by (4.18), let

$$
A(\varepsilon)=\bigcup_{i \in \mathbb{N}}(i, i+\varepsilon) \quad(\varepsilon \in[0,1]) .
$$

Then

$$
\lim _{s \rightarrow 1} P_{S}(A(\varepsilon))=\varepsilon
$$

Proof. From (4.18), we have

$$
P_{s}(A)=\sum_{i=1}^{\infty}\left(\frac{\Theta_{1}^{\lambda}(1, s, i ; b)-\Theta_{1}^{\lambda}(1, s, i+\varepsilon ; b)}{\Theta_{1}^{\lambda}(1, s, 1 ; b)}\right) .
$$

By expanding the function $\Theta_{1}^{\lambda}(1, s, i+\varepsilon, b)$ by means of Taylor's series and using the derivative formula (4.11), we get

$$
\begin{aligned}
P_{s}(A) & =\frac{1}{\Theta_{1}^{\lambda}(1, s, 1 ; b)} \\
\cdot(\varepsilon s & \sum_{i=1}^{\infty} \Theta_{1}^{\lambda}(1, s+1, i ; b) \\
& \left.\quad-\frac{\varepsilon^{2}}{2} s(s+1) \sum_{i=1}^{\infty} \Theta_{1}^{\lambda}(1, s+2, i ; b)+\cdots\right)
\end{aligned}
$$

We now consider each sum in (4.22) separately. We thus find that

$$
\begin{aligned}
& \sum_{i=1}^{\infty} \Theta_{1}^{\lambda}(1, s+m, i ; b)=\frac{1}{\lambda \Gamma(s+m)} \\
& \cdot \sum_{i=1}^{\infty} \sum_{n=0}^{\infty} \frac{H_{0,2}^{2,0}\left[(i+n) b^{\frac{1}{\lambda}} \mid \overline{(s+m, 1),\left(0, \frac{1}{\lambda}\right)}\right]}{(i+n)^{s+m}} \\
& =\frac{1}{\lambda \Gamma(s+m)} \\
& \cdot \sum_{j=0}^{\infty} \sum_{n=0}^{\infty} \frac{H_{0,2}^{2,0}\left[(j+n+1) b^{\frac{1}{\lambda}} \mid \overline{\left.(s+m, 1),\left(0, \frac{1}{\lambda}\right)\right]}\right.}{(j+n+1)^{s+m}} .
\end{aligned}
$$

Since the number of non-negative integer solutions of the Diophantine equation $j+n=N$ is

$$
\left(\begin{array}{c}
N+1 \\
1
\end{array}\right)=N+1
$$

the double summation in (4.23) can be replaced by a single summation, that is,

$$
\begin{aligned}
& \sum_{i=1}^{\infty} \Theta_{1}^{\lambda}(1, s+m, i ; b) \\
& \quad=\frac{1}{\lambda \Gamma(s+m)} \sum_{N=0}^{\infty} \frac{H_{0,2}^{2,0}\left[(N+1) b^{\frac{1}{\lambda}} \mid \overline{(s+m, 1),\left(0, \frac{1}{\lambda}\right)}\right]}{(N+1)^{s+m-1}} \\
& =\Theta_{1}^{\lambda}(1, s+m-1,1 ; b) .
\end{aligned}
$$

We thus obtain

$$
\begin{aligned}
& \lim _{s \rightarrow 1} P_{S}(A) \\
& =\lim _{s \rightarrow 1}\left(\varepsilon s \frac{\Theta_{1}^{\lambda}(1, s, 1 ; b)}{\Theta_{1}^{\lambda}(1, s, 1 ; b)}-\frac{\varepsilon^{2}}{2} s(s+1) \frac{\Theta_{1}^{\lambda}(1, s+1,1 ; b)}{\Theta_{1}^{\lambda}(1, s, 1 ; b)}+\cdots\right) \\
& =\varepsilon-\frac{\varepsilon^{2}}{2} s(s+1) \lim _{s \rightarrow 1} \frac{\Theta_{1}^{\lambda}(1, s+1,1 ; b)}{\Theta_{1}^{\lambda}(1, s, 1 ; b)}+\cdots
\end{aligned}
$$


We note that, when $s \rightarrow 1$, the series for $\Theta_{1}^{\lambda}(1, s, 1 ; b)$ is divergent and the series for $\Theta_{1}^{\lambda}(1, s+1,1 ; b)$ is convergent. Therefore, all other terms vanish in (4.25) except the leading term. Consequently, we get

$$
\lim _{s \rightarrow 1} P_{S}(A)=\varepsilon
$$

which completes the proof of Theorem 8 .

It does not seem to be difficult to extend the above-detailed investigation of the generalized Hurwitz measure, which was presented earlier by Srivastava et al. [34], to analogously cover wider and more general situations involving the Hurwitz-Lerch zeta function

$$
\Phi_{\lambda_{1}, \cdots, \lambda_{p} ; \mu_{1}, \cdots, \mu_{q}}^{\left(\rho_{1}, \cdots, \rho_{p}, \sigma_{1}, \cdots, \sigma_{q}\right)}(z, s, a ; b, \lambda)
$$

defined by (1.14). Nevertheless, we choose to turn instead toward an investigation of the following general probability distribution involving this generalized Hurwitz-Lerch zeta function.

Definition 3. A random variable $\xi$ is said to be generalized Hurwitz distributed if its probability density function is given by

$f_{\xi}(a):= \begin{cases}\frac{s \Phi_{\lambda_{1}, \cdots, \lambda_{p}, \mu_{1}, \cdots, \mu_{q}}^{\left(\rho_{1}, \cdots, \rho_{p}, \sigma_{1}, \cdots, \sigma_{q}\right)}(z, s+1, a ; b, \lambda)}{\Phi_{\lambda_{1}, \cdots, \lambda_{p} ; \mu_{1}, \cdots, \mu_{q}}^{\left(\rho_{1}, \cdots, \rho_{p}, \sigma_{1}, \cdots, \sigma_{q}\right)}(z, s, 1 ; b, \lambda)} & (a \geqq 1) \\ 0 & \text { (otherwise), }\end{cases}$

where it is tacitly assumed that the arguments $z, s, b, \lambda$ and $\mu$. and the parameters

$$
\lambda_{j}, \rho_{j} \quad(j=1, \cdots, p) \text { and } \mu_{j}, \sigma_{j} \quad(j=1, \cdots, q),
$$

are fixed and suitably constrained so that the probability density function $f_{\xi}(a)$ remains nonnegative.

Theorem 9. Suppose that $\xi$ is a continuous random variable $\xi$ with its probability density function defined by (4.27). Then the moment generating function $M(\mathfrak{z})$ of the random variable $\xi$ is given by

$$
M(\mathfrak{z}):=\mathbb{E}_{S}\left[e^{\mathfrak{z}} \xi\right]=\sum_{n=0}^{\infty} \mathbb{E}_{S}\left[\xi^{n}\right] \frac{\mathfrak{z}^{n}}{n !}
$$

with the moment $\mathbb{E}_{s}\left[\xi^{n}\right]$ of order $n$ given by

$$
\begin{aligned}
\mathbb{E}_{s}\left[\xi^{n}\right]=\sum_{k=0}^{n} \frac{n !}{(n-k) !} \frac{\Gamma(s-k)}{\Gamma(s)} \\
\cdot \frac{\Phi_{\left.\lambda_{1}, \cdots, \lambda_{p}, \mu_{1}, \cdots, \mu_{q}\right)}^{\left(\rho_{1}, \cdots, \rho_{p}, \sigma_{1}, \cdots, \sigma_{q}\right)}(z, s, 1 ; b, \lambda)}{\Phi_{\lambda_{1}, \cdots, \lambda_{p} ; \mu_{1}, \cdots, \mu_{q}}^{\left(\rho_{1}, \cdots, \rho_{1}, \sigma_{1}, \cdots, \sigma_{q}\right)}(z, s, 1 ; b, \lambda)} .
\end{aligned}
$$

Proof. The assertion in (4.28) can be derived easily by using the exponential series for $e^{\mathfrak{z}} \xi$. On the other hand, since

$$
\begin{aligned}
\frac{\mathrm{d}}{\mathrm{d} a}\{ & \left.\Phi_{\lambda_{1}, \cdots, \lambda_{p} ; \mu_{1}, \cdots, \mu_{q}}^{\left(\rho_{1}, \cdots, \rho_{p}, \sigma_{1}, \cdots, \sigma_{q}\right)}(z, s, a ; b, \lambda)\right\} \\
& =-s \Phi_{\lambda_{1}, \cdots, \lambda_{p} ; \mu_{1}, \cdots, \mu_{q}}^{\left(\rho_{1}, \cdots, \rho_{p}, \sigma_{1}, \cdots, \sigma_{q}\right)}(z, s+1, a ; b, \lambda),
\end{aligned}
$$

which follows readily from the definition (1.14), if we make use of integration by parts, we find from the definition of the moment $\mathbb{E}_{S}\left[\xi^{n}\right]$ that

$$
\begin{aligned}
& \mathbb{E}_{s}\left[\xi^{n}\right]=\int_{1}^{\infty} a^{n} f_{\xi}(a) \mathrm{d} a \\
& =\frac{s}{\Phi_{\lambda_{1}, \cdots, \lambda_{p} ; \mu_{1}, \cdots, \mu_{q}}^{\left(\rho_{1}, \cdots, \rho_{p}, \sigma_{1}, \cdots, \sigma_{q}\right)}(z, s, 1 ; b, \lambda)} \\
& \cdot \int_{1}^{\infty} a^{n} \Phi_{\lambda_{1}, \cdots, \lambda_{p} ; \mu_{1}, \cdots, \mu_{q}}^{\left(\rho_{1}, \cdots, \rho_{p}, \sigma_{1}, \cdots, \sigma_{q}\right)}(z, s+1, a ; b, \lambda) \mathrm{d} a \\
& =-\frac{1}{\Phi_{\lambda_{1}, \cdots, \lambda_{p} ; \mu_{1}, \cdots, \mu_{q}}^{\left(\rho_{1}, \cdots, \rho_{p}, \sigma_{1}, \cdots, \sigma_{q}\right)}(z, s, 1 ; b, \lambda)} \\
& \cdot \int_{1}^{\infty} a^{n} \frac{\mathrm{d}}{\mathrm{d} a}\left\{\Phi_{\lambda_{1}, \cdots, \lambda_{p} ; \mu_{1}, \cdots, \mu_{q}}^{\left(\rho_{1}, \cdots, \rho_{p}, \sigma_{1}, \cdots, \sigma_{q}\right)}(z, s, a ; b, \lambda)\right\} \mathrm{d} a \\
& =-\left.\frac{a^{n} \Phi_{\lambda_{1}, \cdots, \lambda_{p}, \mu_{1}, \cdots, \mu_{q}}^{\left(\rho_{1}, \cdots, \rho_{p}, \sigma_{1}, \cdots, \sigma_{q}\right)}(z, s, a ; b, \lambda)}{\Phi_{\lambda_{1}, \cdots, \lambda_{p}, \mu_{1}, \cdots, \mu_{q}}^{\left(\rho_{1}, \cdots, \sigma_{q}, \cdots, \sigma_{q}\right)}(z, s, 1 ; b, \lambda)}\right|_{a=1} ^{\infty} \\
& +\frac{n}{\Phi_{\lambda_{1}, \cdots, \lambda_{p} ; \mu_{1}, \cdots, \mu_{q}}^{\left(\rho_{1}, \cdots, \rho_{p}, \sigma_{1}, \cdots, \sigma_{q}\right)}(z, s, 1 ; b, \lambda)} \\
& \cdot \int_{1}^{\infty} a^{n-1} \Phi_{\lambda_{1}, \cdots, \lambda_{p} ; \mu_{1}, \cdots, \mu_{q}}^{\left(\rho_{1}, \cdots, \rho_{p}, \sigma_{1}, \cdots, \sigma_{q}\right)}(z, s, a ; b, \lambda) \mathrm{d} a \\
& =1-\lim _{a \rightarrow \infty}\left\{\frac{a^{n} \Phi_{\lambda_{1}, \cdots, \lambda_{p} ; \mu_{1}, \cdots, \mu_{q}}^{\left(\rho_{1}, \cdots, \rho_{p}, \sigma_{1}, \cdots, \sigma_{q}\right)}(z, s, a ; b, \lambda)}{\Phi_{\lambda_{1}, \cdots, \lambda_{p} ; \mu_{1}, \cdots, \mu_{q}}^{\left(\rho_{1}, \cdots, \rho_{p}, \sigma_{1}, \cdots, \sigma_{q}\right)}(z, s, 1 ; b, \lambda)}\right\} \\
& +\frac{n}{\Phi_{\lambda_{1}, \cdots, \lambda_{p} ; \mu_{1}, \cdots, \mu_{q}}^{\left(\rho_{1}, \cdots, \rho_{q}, \sigma_{1}, \cdots, \sigma_{q}\right)}(z, s, 1 ; b, \lambda)} \\
& \cdot \int_{1}^{\infty} a^{n-1} \Phi_{\lambda_{1}, \cdots, \lambda_{p} ; \mu_{1}, \cdots, \mu_{q}}^{\left(\rho_{1}, \cdots, \rho_{p}, \sigma_{1}, \cdots, \sigma_{q}\right)}(z, s, a ; b, \lambda) \mathrm{d} a \\
& =1+\frac{n}{\Phi_{\lambda_{1}, \cdots, \lambda_{p} ; \mu_{1}, \cdots, \mu_{q}}^{\left(\rho_{1}, \cdots, \rho_{p}, \sigma_{1}, \cdots, \sigma_{q}\right)}(z, s, 1 ; b, \lambda)} \\
& \cdot \int_{1}^{\infty} a^{n-1} \Phi_{\lambda_{1}, \cdots, \lambda_{p} ; \mu_{1}, \cdots, \mu_{q}}^{\left(\rho_{1}, \cdots, \rho_{p}, \sigma_{1}, \cdots, \sigma_{q}\right)}(z, s, a ; b, \lambda) \mathrm{d} a
\end{aligned}
$$$$
(n \in \mathbb{N}) \text {, }
$$

where, in addition to the derivative property (4.30), we have used the following limit formula:

$$
\begin{aligned}
\lim _{a \rightarrow \infty}\left\{a^{n} \Phi_{\lambda_{1}, \cdots, \lambda_{p} ; \mu_{1}, \cdots, \mu_{q}}^{\left(\rho_{1}, \cdots, \rho_{p}, \sigma_{1}, \cdots, \sigma_{q}\right)}(z, s, a ; b, \lambda)\right\} \\
=\lim _{a \rightarrow \infty}\left\{\frac{a^{n}}{\Gamma(s)} \int_{0}^{\infty} t^{s-1} \exp \left(-a t-\frac{b}{t^{\lambda}}\right)\right\} \mathrm{d} t \\
=\frac{1}{\Gamma(s)} \int_{0}^{\infty} t^{s-1} \exp \left(-\frac{b}{t^{\lambda}}\right) \lim _{a \rightarrow \infty}\left\{a^{n} e^{-a t}\right\} \\
\cdot{ }_{p} \Psi_{q}^{*}\left[\begin{array}{l}
\left(\lambda_{1}, \rho_{1}\right), \cdots,\left(\lambda_{p}, \rho_{p}\right) ; \\
\left(\mu_{1}, \sigma_{1}\right), \cdots,\left(\mu_{q}, \sigma_{q}\right) ;
\end{array}\right] \mathrm{d} t
\end{aligned}
$$




$$
=0 \quad(n \in \mathbb{N}) .
$$

Consequently, we have the following reduction formula for $\mathbb{E}_{S}\left[\xi^{n}\right]$ :

$$
\begin{array}{r}
\mathbb{E}_{s}\left[\xi^{n}\right]=1+\frac{\Phi_{\lambda_{1}, \cdots, \lambda_{p} ; \mu_{1}, \cdots, \mu_{q}}^{\left(\rho_{1}, \cdots, \rho_{p}, \sigma_{1}, \cdots, \sigma_{q}\right)}(z, s-1,1 ; b, \lambda)}{\Phi_{\lambda_{1}, \cdots, \lambda_{p} ; \mu_{1}, \cdots, \mu_{q}}^{\left(\rho_{1}, \cdots, \rho_{p}, \sigma_{1}, \cdots, \sigma_{q}\right)}(z, s, 1 ; b, \lambda)} \\
\cdot \frac{n}{s-1} \mathbb{E}_{s-1}\left[\xi^{n-1}\right] \quad(n \in \mathbb{N}) .
\end{array}
$$

By iterating the recurrence (4.31), we arrive at the desired result (4.29) asserted by Theorem 6.

Remark 9. A special case of Theorem 6 when

$$
p-1=q=0, \quad \lambda_{1}=\mu=1 \quad \text { and } \quad \rho_{1}=1
$$

was considered by Srivastava et al. [34]. Moreover, in an earlier investigation, Gupta et al. [12] considered some particularly simple forms of the Hurwitz-Lerch zeta distributions and their applications in reliability theory. On the other hand, in a very recent investigation, Saxena et al. [25] made use of some specialized cases of the extended Hurwitz-Lerch zeta function

$$
\Phi_{\lambda_{1}, \cdots, \lambda_{p} ; \mu_{1}, \cdots, \mu_{q}}^{\left(\rho_{1}, \cdots, \rho_{p}, \sigma_{1}, \cdots, \sigma_{q}\right)}(z, s, a)
$$

of Srivastava et al. [36, p. 503, Equation (6.2)] (see also [27] and [32]), which is defined here by (1.10), in statistical inference.

\section{References}

[1] M. Abramowitz and I. A. Stegun (Editors), Handbook of Mathematical Functions with Formulas, Graphs, and Mathematical Tables, Applied Mathematics Series, National Bureau of Standards, Washington, D.C., 55, 1964; Reprinted by Dover Publications, New York, (1965) (see also [22]).

[2] A. Bayad and J. Chikhi, Reduction and duality of the generalized Hurwitz-Lerch zetas, Fixed Point Theory Appl., 2013, Article ID 82, 1-14 (2013).

[3] A. Bayad and S. Gaboury, Generalized Dirichlet $L$-function of arbitrary order with applications, Adv. Stud. Contemp. Math., 23, 607-619 (2013).

[4] M. A. Chaudhry and S. M. Zubair, On a Class of Incomplete Gamma Functions with Applications, Chapman and Hall (CRC Press Company), Boca Raton, London, New York and Washington, D.C., (2001).

[5] A. Erdélyi, W. Magnus, F. Oberhettinger and F. G. Tricomi, Higher Transcendental Functions,, McGraw-Hill Book Company, New York, Toronto and London, I, (1953).

[6] A. Erdélyi, W. Magnus, F. Oberhettinger and F. G. Tricomi, Tables of Integral Transforms, McGraw-Hill Book Company, New York, Toronto and London, II, (1954).

[7] S. Gaboury and A. Bayad, Series representations at special values of generalized Hurwitz-Lerch zeta function, Abstr. Appl. Anal., 2013, Article ID 975615, 1-8 (2013).
[8] M. Garg, K. Jain and S. L. Kalla, A further study of general Hurwitz-Lerch zeta function, Algebras Groups Geom., 25 (2008), 311-319 (2008).

[9] M. Garg, K. Jain and H. M. Srivastava, Some relationships between the generalized Apostol-Bernoulli polynomials and Hurwitz-Lerch Zeta functions, Integral Transforms Spec. Funct., 17 (2006), 803-815.

[10] S. P. Goyal and R. K. Laddha, On the generalized Zeta function and the generalized Lambert function, Ganita Sandesh, 11, 99-108 (1997).

[11] S. W. Golomb, A class of probability distributions on the integers, J. Number Theory, 2, 189-192 (1970).

[12] P. L. Gupta, R. C. Gupta, S.-H. Ong and H. M. Srivastava, A class of Hurwitz-Lerch Zeta distributions and their applications in reliability, Appl. Math. Comput., 196, 521531 (2008).

[13] A. A. Kilbas and M. Saigo, H-Transforms: Theory and Applications, Chapman and Hall (CRC Press Company), Boca Raton, London, New York and Washington, D.C., (2004).

[14] A. A. Kilbas, H. M. Srivastava and J. J. Trujillo, Theory and Applications of Fractional Differential Equations, NorthHolland Mathematical Studies, Vol. 204, Elsevier (NorthHolland) Science Publishers, Amsterdam, London and New York, (2006).

[15] S.-D. Lin and H. M. Srivastava, Some families of the Hurwitz-Lerch Zeta functions and associated fractional derivative and other integral representations, Appl. Math. Comput., 154, 725-733 (2004).

[16] S.-D. Lin, H. M. Srivastava and P.-Y. Wang, Some expansion formulas for a class of generalized Hurwitz-Lerch Zeta functions, Integral Transforms Spec. Funct., 17, 817-827 (2006).

[17] R. A. Lippert, A probabilistic interpretation of the Hurwitz zeta function, Adv. Math., 97, 278-284 (1993).

[18] M.-J. Luo and R. K. Raina, Some new results related to a class of generalized Hurwitz zeta function, Preprint 2013 [Ann. Polon. Math. (submitted for publication)].

[19] A. M. Mathai and R. K. Saxena, The H-Functions with Applications in Statistics and Other Disciplines, Wiley Eastern Limited, New Delhi, (1978).

[20] A. M. Mathai and R.K. Saxena, Generalized Hypergeometric Functions with Applications in Statistics and Physical Sciences, Lecture Notes in Mathematics, Springer-Verlag, Berlin, Heidelberg and New York, 348, (1973).

[21] A. M. Mathai, R. K. Saxena and H. J. Haubold, The HFunction: Theory and Applications, Springer, New York, Dordrecht, Heidelberg and London, (2010).

[22] F. W. J. Olver, D. W. Lozier, R. F. Boisvert and C. W. Clark (Editors), NIST Handbook of Mathematical Functions [With 1 CD-ROM (Windows, Macintosh and UNIX)], U. S. Department of Commerce, National Institute of Standards and Technology, Washington, D. C., (2010); Cambridge University Press, Cambridge, London and New York, (2010) (see also [1]).

[23] R. K. Raina and P. K. Chhajed, Certain results involving a class of functions associated with the Hurwitz zeta function, Acta Math. Univ. Comenianae, 73, 89-100 (2004).

[24] S. G. Samko, A. A. Kilbas and O. I. Marichev, Fractional Integrals and Derivatives: Theory and Applications, 
Translated from the Russian: Integrals and Derivatives of Fractional Order and Some of Their Applications ("Nauka i Tekhnika", Minsk, 1987), Gordon and Breach Science Publishers, Reading, Tokyo, Paris, Berlin and Langhorne (Pennsylvania), (1993).

[25] R. K. Saxena, T. K. Pogány, R. Saxena and D. Jankov, On generalized Hurwitz-Lerch Zeta distributions occurring in statistical inference, Acta Univ. Sapientiae Math., 3, 43-59 (2011).

[26] H. M. Srivastava, Some formulas for the Bernoulli and Euler polynomials at rational arguments, Math. Proc. Cambridge Philos. Soc., 129, 77-84 (2000).

[27] H. M. Srivastava, Some generalizations and basic (or $q$-) extensions of the Bernoulli, Euler and Genocchi polynomials, Appl. Math. Inform. Sci., 5, 390-444 (2011).

[28] H. M. Srivastava, Generating relations and other results associated with some families of the extended HurwitzLerch Zeta functions, SpringerPlus, 2, Article ID 2:67, 1-14 (2013).

[29] H. M. Srivastava and J. Choi, Series Associated with the Zeta and Related Functions, Kluwer Academic Publishers, Dordrecht, Boston and London, (2001).

[30] H. M. Srivastava and J. Choi, Zeta and q-Zeta Functions and Associated Series and Integrals, Elsevier Science Publishers, Amsterdam, London and New York, (2012).

[31] H. M. Srivastava, K. C. Gupta and S. P. Goyal, The HFunctions of One and Two Variables with Applications, South Asian Publishers, New Delhi and Madras, (1982).

[32] H. M. Srivastava, D. Jankov, T. K. Pogány and R. K. Saxena, Two-sided inequalities for the extended Hurwitz-Lerch Zeta function, Comput. Math. Appl., 62, 516-522 (2011).

[33] H. M. Srivastava and P. W. Karlsson, Multiple Gaussian Hypergeometric Series, Halsted Press (Ellis Horwood Limited, Chichester), John Wiley and Sons, New York, Chichester, Brisbane and Toronto, (1985).

[34] H. M. Srivastava, M.-J. Luo and R. K. Raina, New results involving a class of generalized Hurwitz-Lerch zeta functions and their applications, Turkish J. Anal. Number Theory, 1, 26-35 (2013).

[35] H. M. Srivastava and H. L. Manocha, A Treatise on Generating Functions, Halsted Press (Ellis Horwood Limited, Chichester), John Wiley and Sons, New York, Chichester, Brisbane and Toronto, (1984).

[36] H. M. Srivastava, R. K. Saxena, T. K. Pogány and R. Saxena, Integral and computational representations of the extended Hurwitz-Lerch Zeta function, Integral Transforms Spec. Funct., 22, 487-506 (2011).

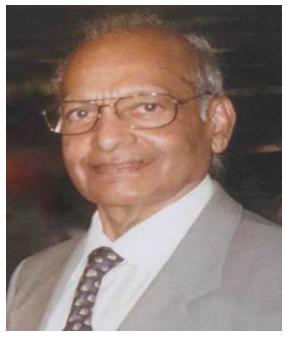

H. M. Srivastava For the author's biographical and other professional details the interested reader should look into the following Web Site:

http://www.math.uvic.ca/faculty/harimsri/ 